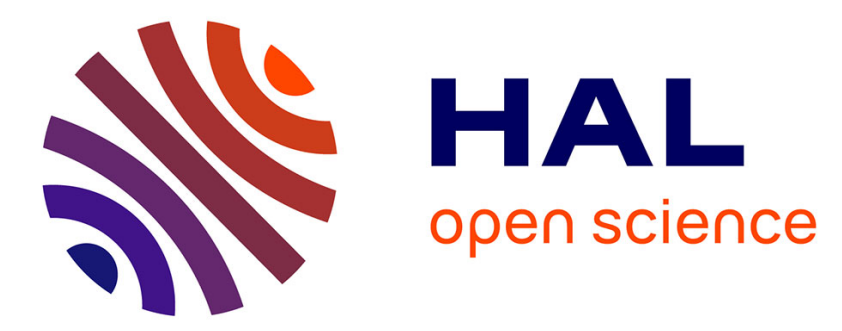

\title{
L'aqueduc d'Antipolis dit de la Bouillide (Alpes-Maritimes)
}

Paul Garczynski, Jean Foucras, Michel Dubar

\section{To cite this version:}

Paul Garczynski, Jean Foucras, Michel Dubar. L'aqueduc d'Antipolis dit de la Bouillide (AlpesMaritimes). Gallia - Archéologie de la France antique, 2005, Aqueducs de la Gaule méditerranéenne, 62, pp.13-34. 10.3406/galia.2005.3218 . hal-01912566

\section{HAL Id: hal-01912566 https://hal.science/hal-01912566}

Submitted on 14 Jan 2020

HAL is a multi-disciplinary open access archive for the deposit and dissemination of scientific research documents, whether they are published or not. The documents may come from teaching and research institutions in France or abroad, or from public or private research centers.
L'archive ouverte pluridisciplinaire HAL, est destinée au dépôt et à la diffusion de documents scientifiques de niveau recherche, publiés ou non, émanant des établissements d'enseignement et de recherche français ou étrangers, des laboratoires publics ou privés.

\section{(ㅇ)(1) $\$$}

Distributed under a Creative Commons Attribution - NonCommercial - NoDerivatives| 4.0 


\title{
L'AQUEDUC D'ANTIPOLIS DIT DE LA BOUILLIDE (ALPES-MARITIMES)
}

\author{
Paul GARCZYNSKI et Jean FOUCRAS avec la collaboration de Michel DUBAR
}

\begin{abstract}
Mots-clés. Pont-aqueduc, archivolte, hydraulique, canal, concrétion, contre-parement.
Résumé. Antipolis aurait eu trois aqueducs dont deux sont encore attestés par des vestiges. Celui qui fait l'objet de cette étude est caractérisé par une branche principale dite de la Bouillide qui lui a donné son appellation actuelle, et une branche secondaire dite de la Valmasque. Les recherches récentes sur le parcours supérieur ont permis de reconnâttre un passage en tunnel, un pont à une arche, effondré, un pont à deux arches et un pont à cinq arches dont il ne reste en élévation que les culées et une pile. À mi-parcours se trouvait un bassin de décantation ou de régulation.

Le conduit ne comporte pas de regard de visite, le canal (0,45 m par 1,20 m sous voûte) ne permettant pas le passage d'un homme. Une similitude architecturale avec l'aqueduc de Fréjus et les thermes de Cimiez laisse supposer qu'il leur est contemporain. Sa durée de fonctionnement, estimée par l'étude des lamines, serait de l'ordre de 160 ans.
\end{abstract}

Key-words. Suspended-aqueduct, archivolt, hydraulics, channel, counter-facing, concretion.

Abstract. It seems that Antipolis had three aqueducts, two of which are known by visible remains. The aqueduct which is studied here is characterized by a main branch "de la Bouillide", name presently given to il, and by a secondary branch " de la Valmasque ". Recent

research on the upper course has allowed to recognize an underground section, a bridge with an arch, now collapsed, a bridge with two arches and another one of which only the culées and a pier of the five arches are still in elevation. A settling or regulation tank was situated half-way:

There was no manhole in the conduit since it was not possible to pass through the channel $(0,45 \mathrm{~m} \times 1,20 \mathrm{~m}$ under the vault). The architectural similarity with the aqueduct of Fréjus and the thermae of Cimiez suggests it was contemporaneous. It must have been in function for about 160 years, from the study of the laminae.

Translation : Isabelle FAUDUET

Schlagwörter. Aquädukt, Vorsatzschale, Wasserbau, Kanal, Ablagerungen, gemauertes Gewölbe.

Zusammenfassung. Zwei der ursprünglich wohl drei römischen Wasserleitungen von Antipolis sind noch durch archäologische Überreste nachweisbar. Die hier untersuchte besteht aus dem Hauptstrang "de la Bouillide", der ihr auch ihren modernen Namen eingetragen hat, und dem Nebenstrang „de la Valmasque“. Neuere Forschungen zum oberen Abschnitt erlauben die Unterscheidung einer durch einen Tunnel geführten Teilstrecke, einer Brücke über einem (heute eingestürzten) Bogen, einer weiteren Brücke über zwei Bögen und schließlich einer Brücke über fünf Bögen, von welchen aber nur noch die äußeren Widerlager und die Pfeiler aufrechtstehen. Auf halbem Wege bestand ein Absetz- oder Regulierungsbecken.

Die Leitung besitzt keine Revisionsschächte, denn der überwölbte Kanal (Querschnitt: 0,45 m Breite $x$ 1,20 m Scheitelhöhe) ist für Personen unpassierbar. Einzig die Ähnlichkeit ihrer Bauweise mit die Wasserleitung von Fréjus und die Bäder von Cimiez führt zu der Annahme, daß die hier untersuchte mit diesen gleichzeitig sein dürfte. Die anhand der Abnutzungsspuren geschätzte Benutzungszeit wird sich wohl auf etwa 160 Jahre belaufen haben.

Übersetzung : Stefan WIRTH

Comme pour bien d'autres agglomérations antiques romanisées, le besoin en eau d'Antipolis a excédé les ressources du site : sources, puits et citernes ${ }^{18}$. Il a donc été nécessaire de capter des sources éloignées et d'amener l'eau en ville grâce

18. Mentionnés dans des écrits mais sans localisation. Pour les citernes, nous en connaissons deux côte à côte, sous la chausséc entre la cathćdrale et le château Grimaldi, et une troisième qui semble médiérale, se à des aqueducs. Pour Antipolis, trois aqueducs sont cités dans les écrits d'érudits locaux, dont deux sont matériellement attestés : celui de Fontrieille et celui de la Bouillide (fig. 2). L'aqueduc de Fontrieille, dont les captages supposés sont

trouvant à l'intérieur de ce dernier; les relevés précis de ces citernes ont ćtć cffectućs cn 1966 par A. Olivicr, qui nous en a aimablement transmis les copies. 


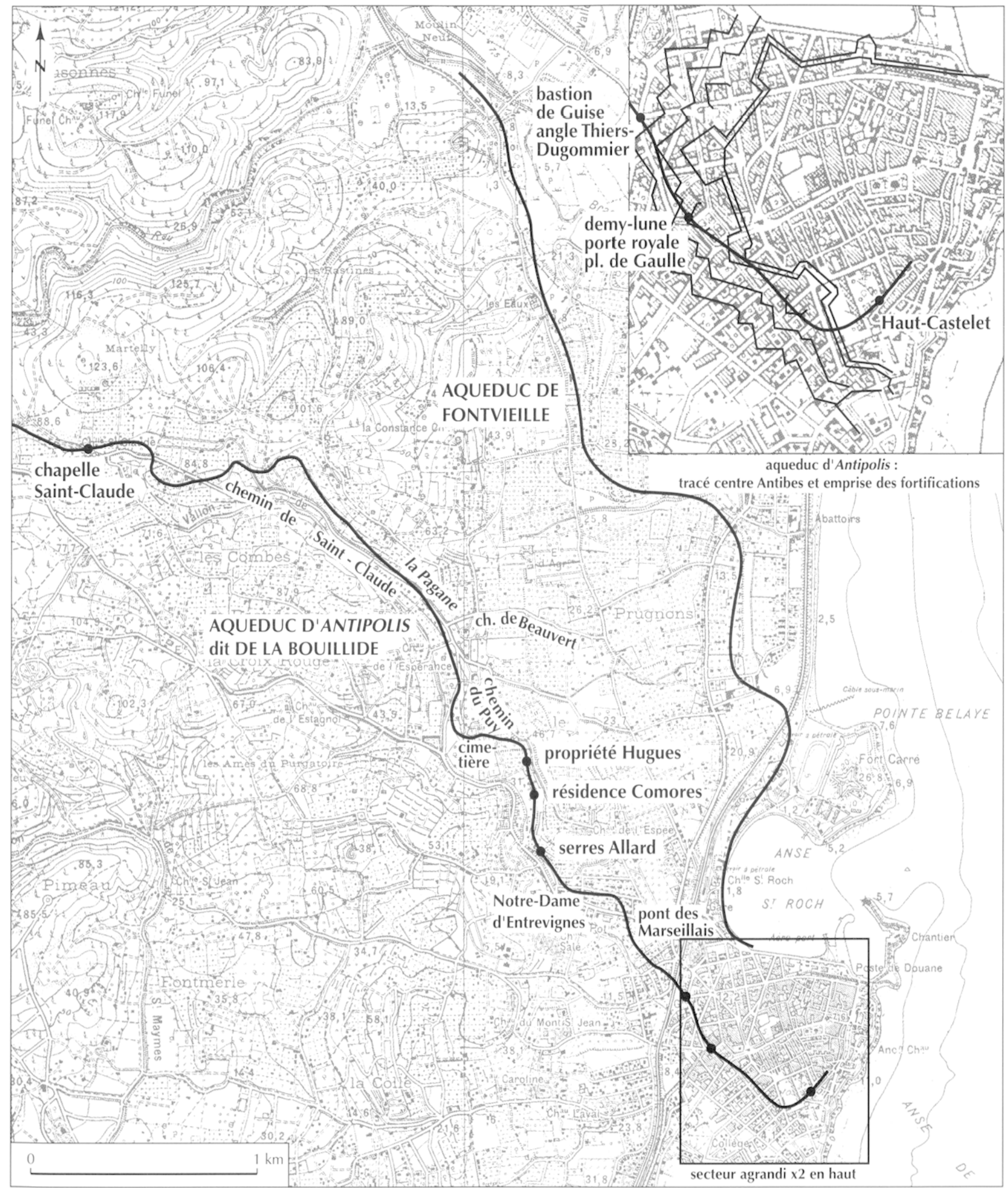

Fig. 2 - I es aqueducs romains d'Antipolis el de Fontrieille dans l'agglomération d'Antibes (fond de carte de l'armé, 1931). Echelle: $1 / 25000$ ).

situés en bordure de la route reliant Antibes à Biot, a été remis en service en 1785 par le colonel d'Aguillon du Corps Royal du Génie, et un tronçon était encore utilisć par la Compagnie des eaux jusquà l'été 2004. L’aqueduc de la Bouillide fait l'objet de cette étude. Un troisième aqueduc est quelquefois cité mais non localisé : il était supposé alimenter les thermes gràce aux caux d'une source situce au nord de l'anse Saint-Roch. 


\section{HISTORIQUE DES RECHERCHES}

Le texte le plus ancien concernant les aqueducs d'Antipolis semble être le manuscrit du chevalier Jean Arazi (1708), recopié à la plume (y compris les dessins) par l'abbé Rostan en 1863. Ce texte collationné, revu, corrigé et annoté, a été ensuite publié (Sardou, Blanc, 1881). Bien que les plus grandes précautions soient à prendre à sa lecture, il est intéressant de noter qu'Arazi attire l'attention sur les destructions que va entraîner la construction des fortifications, et qu'il localise deux sites ainsi appelés à disparaître et relatifs à l'aqueduc dit de la Bouillide (p. 54-56) : "Il y a aussi une issue d'acqueduc sur le chemin royal près du fossé à l'angle du bastion de Guise, une autre à la demy-lune dans l'endroit où l'on fait la porte royale, et un troisiesme vestige dans la ville vers la citadelle ; mais tout cela va estre bientost supprimé par les nouuelles fortifications."

$\mathrm{Au} \mathrm{XIX}^{\mathrm{e}}$ s., plusieurs auteurs ont cité plus ou moins brièvement et avec plus ou moins d'erreurs les aqueducs romains d'Antipolis (Depigny, 1849, p. 11 ; Aubert, Aubert, 1869, p. 33 ; Petit, 1870 ; Tisserand, 1876, p. 42-43; Valcourt, 1878, p. 292-295). Au XXe s., les publications ont été plus nombreuses. On peut les diviser en deux grandes familles : les guides touristiques que nous n'énumérerons pas mais qui présentent souvent un intérêt iconographique, et les textes à caractère technique pour ne pas dire scientifique: Formigé, 1942 (manuscrit, archives municipales d'Antibes) ; Marguier, 1955 ; Raymon, 1955, p. 4-6 ; Grenier, 1960 ; Clergues, 1966 ; Compan, 1980, p. 43-44 et 1987; Cosson, 1989 et 1995; Morena, Counord, 1994, p. 47-51 ; Violino, 1985.

A. Grenier, dans son Manuel d'archéologie gallo-romaine (Grenier, 1960), fait passer le canal de l'aqueduc dit des Clausonnes (aujourd'hui dit de la Bouillide) aux Encourdoules (Vallauris), au voisinage de la Cabre d'Or. Or, ce monument funéraire est situé sur la commune de Biot, et l'aqueduc n'a aucune relation géographique avec l'un ou l'autre de ces deux sites; il y a bien une Chèvre d'Or à Vallauris mais il s'agit d'un aven. La plupart des autres documents cités contiennent également des erreurs plus d'observation que d'interprétation.

Finalement les textes les plus sérieux sont ceux de J. Marguier et de P. Cosson. J. Marguier est le premier à publier une carte, qu'il accompagne de photos des ponts et ses descriptions sont assez fidèles malgré des erreurs; il est aussi le premier à parler de la branche de la Valmasque avec illustrations à l'appui. P. Cosson a précisé et développé le travail de Marguier, signalant aussi que depuis: "l'évolution démographique d'Antibes et de Sophia Antipolis a fait disparaitre de nombreux vestiges de l'ancien aqueduc ". Il donne des indications de sites en zone urbaine dont plusieurs ont pu être vérifiés; d'autres ont disparu mais la précision des descriptions et localisations nous permet de les considérer comme vraisemblables.

Ces publications et les témoignages recueillis dès le début de cette étude ont servi de base à nos travaux de terrain, prospections et sondages. Les hypothèses de tracé pour les tronçons inconnus s'appuient, elles, sur des cartes antérieures au développement urbain et à la construction de l'autoroute A8 dans les années 1960. Certaines découvertes qui ont permis de préciser ce tracé sont le résultat d'informations récoltées à l'occasion de contacts fortuits ou rolontaires.

Au ru des connaissances antérieures, l'aqueduc était caractérisé par une branche principale dite de la Bouillide à laquelle se serait raccordée plus tard la branche dite de la Valmasque (fig. 3). La première appellation a subsisté pour désigner l'ensemble de l'aqueduc, sans doute en raison de sa plus grande longueur. De la branche de la Valmasque n'avait été vu qu'un tronçon taillé à flanc de rocher, signalé par J. Marguier et sans doute disparu. Elle a surtout été dans sa plus grande partie détruite par la construction de l'autoroute A8. D'après leur travaux les auteurs fondent l'hypothèse plus vraisemblable que la branche de la Valmasque est soit contemporaine, soit précède juste la branche de la Bouillide, notamment au regard de son tracé (voir infra).

\section{CONTRAINTES ET STRATÉGIE DE TRACÉ}

En considérant la branche de la Bouillide et la branche commune, seules suffisamment connues, on peut compter de la source à Antipolis ( $16 \mathrm{~km}$ environ) au moins quatre unités géographiques principales : (1) vallon de la Bouillide, (2) colline du Fugueiret, (3) colline des Croûtons, ( ).., (4) vallon du Laval, ( )... ; les points de suspension indiquent deux unités possibles mais indéterminables, où le tracé de l'aqueduc reste hypothétique, du fait de la transformation profonde du terrain (entre Croûtons et Laval) ou de la densité immobilière (agglomération d'Antibes). Les contraintes à prendre en compte pour établir le tracé, autrement dit les obstacles à franchir, se situent en fait aux passages d'une unité géographique à l'autre : (1-2) de la Bouillide au Fugueiret : ligne de crête et vallon du Fugueiret; (2-3) du Fugueiret aux Croûtons: vallon de la Valmasque et de son affluent le Goa ; des Croûtons au Laval : inconnu; du Laval à l'arrivée : inconnu.

(1) Vallon de la Bouillide : le canal suit une pente régulière en épousant le relief. Là où le rocher est abrupt, il empêchait la réalisation en sous-sol du canal ; celui-ci est alors taillé dans le rocher, en ménageant une banquette suffisamment large pour les lits d'attente de la voûte.

(1-2) De la Bouillide au Fugueiret : là se trouve la première stratégie particulière. Pour économiser $3350 \mathrm{~m}$ de contournement d'une crête, celle-ci est traversée par un tunnel de $150 \mathrm{~m}$ de long environ. Dès la fin de ce tunnel, se trouve une nouvelle difficulté : un vallon à contourner sur $2300 \mathrm{~m}$, alors que la dénivellation est faible et qu'au fond coule un petit cours d'eau : la stratégie est alors de descendre perpendiculairement aux courbes de niveau. Aucune trace d'ouvrage de franchissement n'ayant subsisté, le cours d'eau fut sans doute traversé directement sans construction particulière.

(2) La colline du Fugueiret est contournée sur toute sa moitié ouest jusqu'à la rivière Valmasque sans contrainte particulière, avec un tracé qui suit sensiblement les courbes de niveau et le franchissement d'un petit vallon par un pont à une arche.

(2-3) Du Fugueiret aux Croûtons : pour atteindre la colline des Croûtons, la branche de la Bouillide ne traverse pas la vallée au plus court, ce qui aurait nécessité un ourrage de plus de 


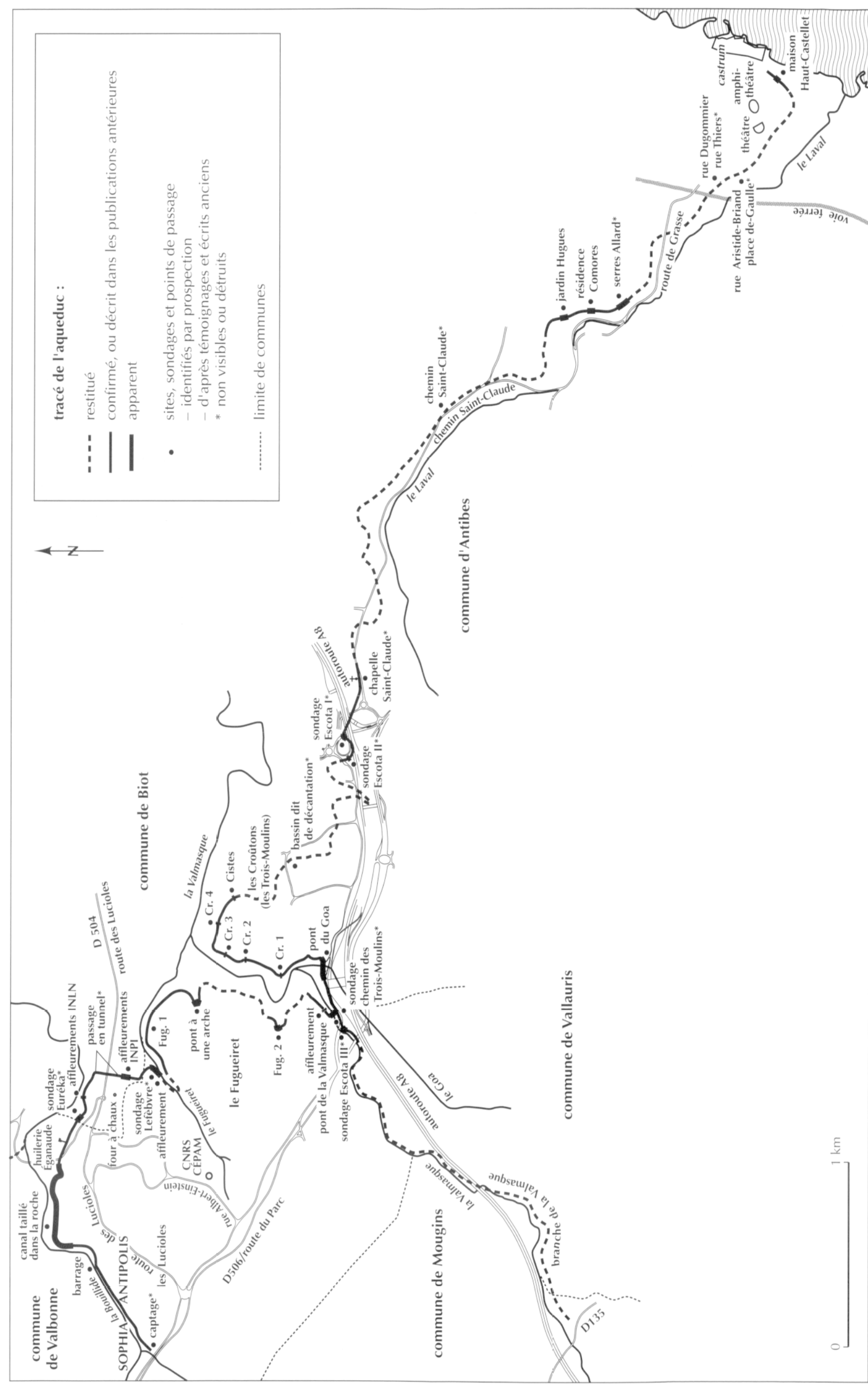

| 
$220 \mathrm{~m}$, mais fait un détour comprenant la réalisation d'un pont sur la Valmasque à la sortie duquel se situe le raccordement avec l'autre branche, et d'un pont sur le Goa, l'ensemble totalisant $2000 \mathrm{~m}$ de canal enterré et $150 \mathrm{~m}$ de ponts. Ce détour suggère que le tracé de la branche de la Bouillide a été conçu à partir de celui de la Valmasque ; deux autres éléments d'observation le laissent également supposer (voir infra, p. 24 et 34).

(3) Colline des Croûtons: comme au Fugueiret le canal enterré suit les courbes de niveau avec sa pente régulière. Un bassin de décantation ou de régulation se situait à ce niveau, approximativement à mi-parcours de l'aqueduc.

(4) À partir de là, les différents sondages et sites observés jusqu'à Antibes ne permettent pas la reconnaissance de tronçons importants de l'aqueduc, ni donc la restitution des contraintes éventuelles auxquels les constructeurs auraient pu faire face.

\section{LA BRANCHE DE LA BOUILLIDE}

\section{LE VALLON DE LA BOUILLIDE}

\section{CAPTAGE}

La zone de captage proposée par les auteurs précédents est située à $128 \mathrm{~m}$ environ d'altitude, en bordure de la route d'Antibes à Valbonne. L'état actuel de cette zone, jardin d'un restaurant d'ailleurs baptisé La Source, ne nous a pas permis la moindre observation permettant de valider cette hypothèse. Seuls deux éléments peuvent la conforter : la présence effective d'une source, et celle, il y a quelques années, d'un bassin, maintenant détruit, qui aurait peut-être constitué un élément de l'installation de captage.

\section{TRACÉ DE CANAL ET BARRAGE TARDIF}

Plus loin, en rive droite de la Bouillide et sur quelques dizaines de mètres, une banquette prolongée par un talus constitue une trace du canal. À l'endroit où ce talus se rapproche du cours d'eau, les fondations d'un barrage sont visibles dans le lit du ruisseau et se poursuivent quelques mètres sur la rive droite. Il s'agit très vraisemblablement du captage des eaux de la Bouillide qui ont alimenté, peut-être en s'ajoutant à celle de la source, le moulin à huile de l'Éganaude à une période récente.

\section{FOSSÉ ET CANAL TAILLÉ DANS LA ROCHE}

Ce tronçon correspond à la partie de l'aqueduc réutilisée comme béal entre le barrage et le moulin ; il forme encore une parcelle sur le cadastre (fig. 4). Le canal romain, dont la voûte a disparu, est bien visible : il se présente comme un fossé sur un replat qui peut atteindre $2 \mathrm{~m}$ de large. En plusieurs endroits, le banc rocheux a été taillé par les constructeurs romains au profil du canal. La partie supérieure du substrat qui correspond au sommet du piédroit a été aplanie, formant un lit d'attente pour la voûte. Là où la roche manque, des assises de moellons maçonnés complètent le piédroit et permettent d'atteindre ce niveau.

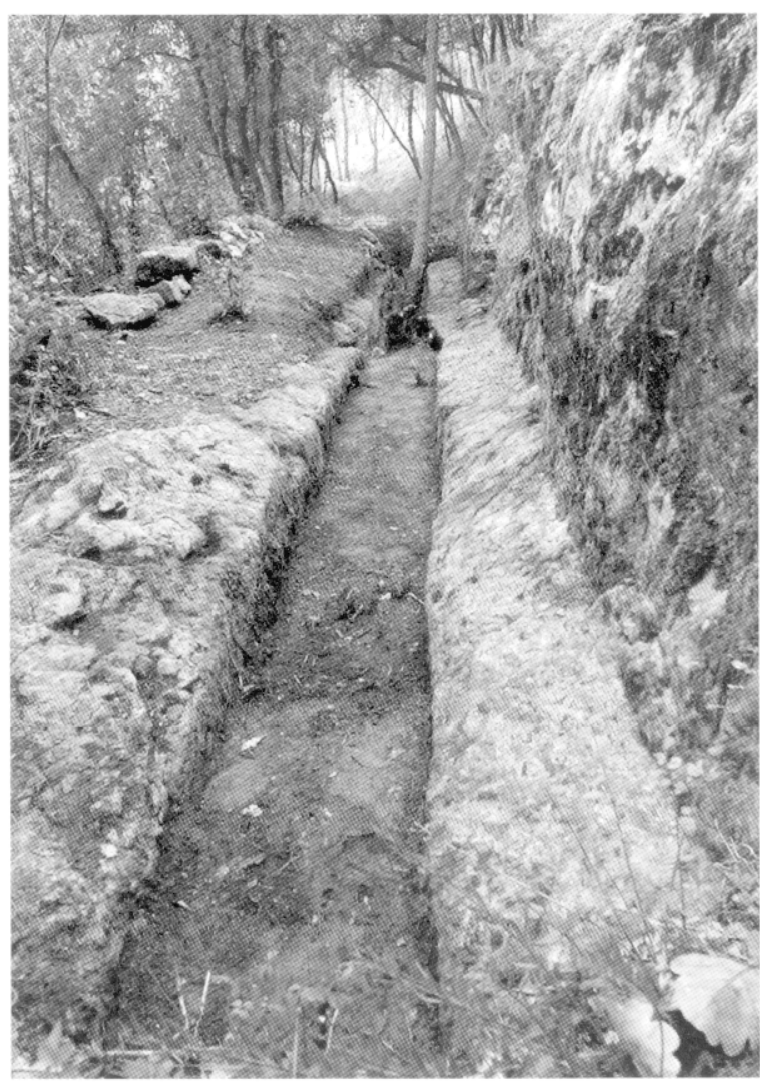

Fig. 4 - Aqueduc d'Antipolis, vallon de la Bouillide: canal taillé dans la roche (cliché P. Garczynski, CNRS).

\section{LE MOULIN DE L'ÉGANAUDE}

Les vestiges de ce moulin à huile ont été préscrvés lors de la construction de l'hôtel Ibis ${ }^{19}$.

\section{LE SITE EURÉKA (IMMEUBLE SOLARIS)}

A Biot, les travaux d'excavation d'un terrain pour la construction d'un immeuble ont entraîné la destruction du canal sur une quinzaine de mètres (fig. 5). En contrepartic, ccla nous a permis l'étude d'une coupe particulièrement intéressante du canal qui était intact et se présentait dans son état en fin d'utilisation (fig. 6).

Le bâti présente quelques particularités : le fond du canal est un bloc de mortier en U coulé directement dans la tranchéc de fondation taillée dans la roche, avec un coffrage pour le conduit. Le liant est fait de chaux et les agrégats sont constitués de calcaire concassé, de dimensions irrégulières $(1,5$ à $8 \mathrm{~cm})$ avec une absence quasi totale de tuileaux. Les sommets

19. Son implantation a été déplacée de $1 \mathrm{~m}$ à la demande du Centre de recherches archéologiques. Quelques éléments (meules et bases de pressoirs) ont été transportés et déposés place Sophie-Laffitte, où ils sont toujours visibles. Mis à part un relevé (Carrier, (arronnski, 1982), il n'y a pas eu d'étude archéologique de ce moulin et le projet de sauvetage des vestiges et de présentation au public n'a pas cu de suite, faute de financement. 
des branches du $\mathrm{C}$ comportent un radier de mortier de $5 \mathrm{~cm}$ d'épaisseur en moyenne. Ce mortier, contrairement au précédent, est d'une grande dureté et d'une grande cohésion. Il sert de base aux piédroits en petit appareil, constitués de quatre rangs de moellons côté canal et d'un blocage

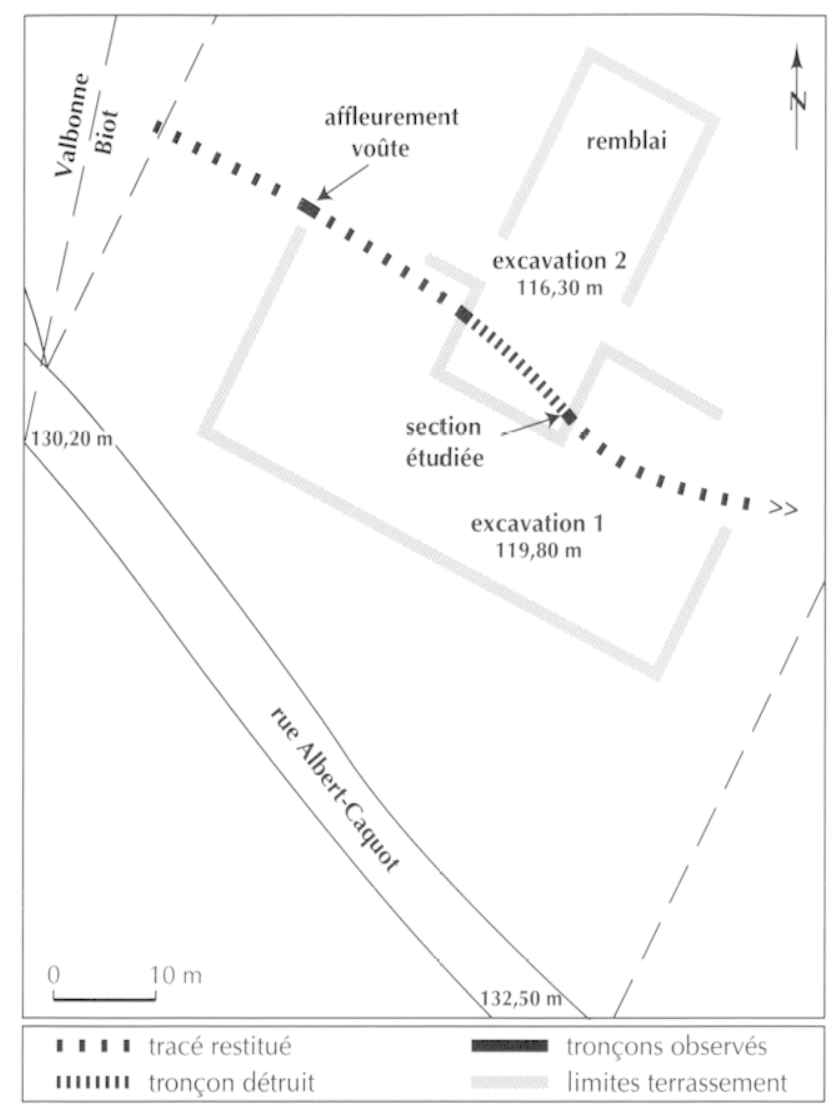

Fig. 5 - Aqueduc d'Antipolis, vallon de la Bouillide: plan du chantier Euréka (DAO P. Garczynski, CNRS).

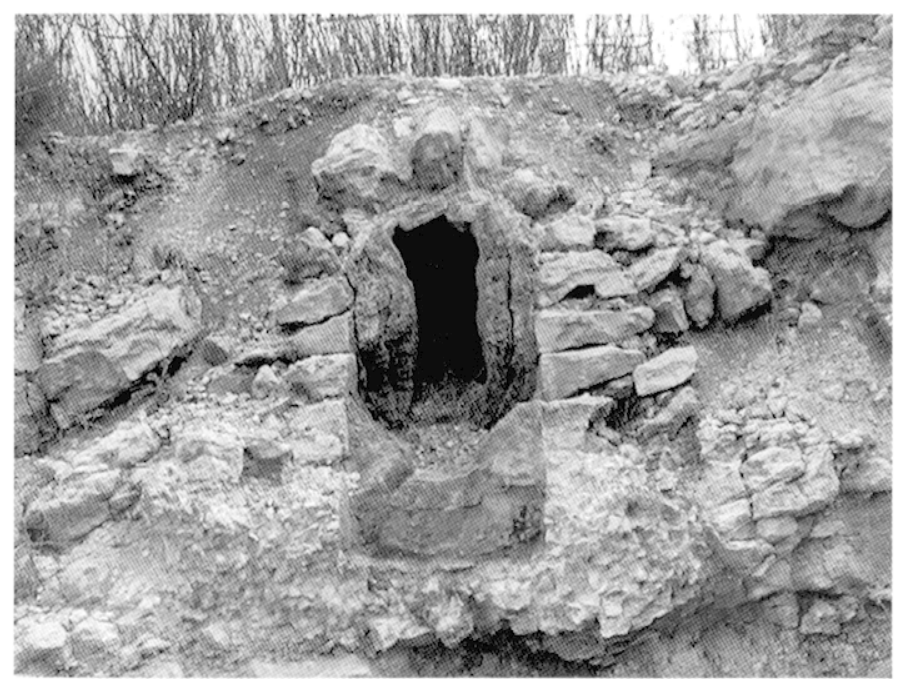

irrégulier côté externe. La voûte est sommairement clavée. Si le mortier d'étanchéité est absent sur la face interne des piédroits, une couche d'étanchéité soignée recouvre l'extrados de la voûte.

Le système concrétionnaire interne, très important, est composé des deux types connus: celui dit en gobelet ou en calice et celui dit en ogive. La section de la veine fluide à la mise en eau (écoulement libre), déterminée grâce à la hauteur de la première lamine déposée, est de $6,75 \mathrm{dm}^{2}(45 \mathrm{~cm} \times 15 \mathrm{~cm})$. À la fin de l'utilisation de l'aqueduc, cette section circonscrite par la dernière lamine déposée de type ogive est de $11 \mathrm{dm}^{2}$. Ce tronçon de canal a donc fonctionné en conduite forcée sans qu'il soit possible d'en tirer une conclusion quant à la vitesse d'écoulement, donc à l'évolution du débit.

\section{CNRS-INLN}

Les vestiges du canal, privé de sa voûte, sont visibles sur une banquette rocheuse dominant le lit de la Bouillide. Il s'agit des derniers vestiges visibles dans ce bassin versant. Sur le site GSF voisin, les derniers mètres avant le passage en tunnel ont été détruits et l'entrée n'a pas été retrouvée, ce secteur ayant fait l'objet de terrassements importants.

\section{DE LA BOUILLIDE AU FUGUEIRET}

\section{LE TUNNEL SOUS LA ROUTE DES LUCIOLES ET LA DESCENTE INPI}

Ces sites illustrent deux des techniques mises en cuvre par les architectes romains pour raccourcir le tracé.

Il s'agit d'abord d'éviter le contournement d'une colline par un franchissement en tunnel sur $150 \mathrm{~m}$ environ de la ligne de crête séparant les bassins versants de la Bouillide et du Fugueiret; une tranchée profonde remblayée récemment (hypothèse de P. Cosson) est peu probable, car elle aurait laissé des traces sur le terrain et serait visible sur les photos aériennes

Fig. 6 - Aqueduc d'Antipolis, vallon de la Bouillide, chantier Euróka : coupe du canal maintenant sonus l'immeuble Solaris (cliché et DAO P. Garczynski et J. Foucras, ('NRS). 


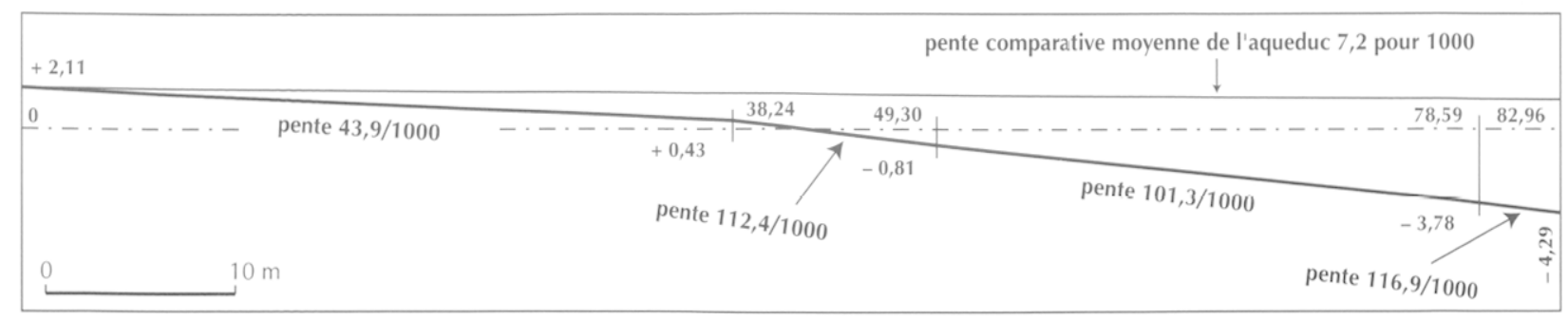

Fig. 7 - Aqueduc d'Antipolis, coupe schématique et pentes de la descente INPI (DAO P. Garczynski et J. Foucras, (XNRS).

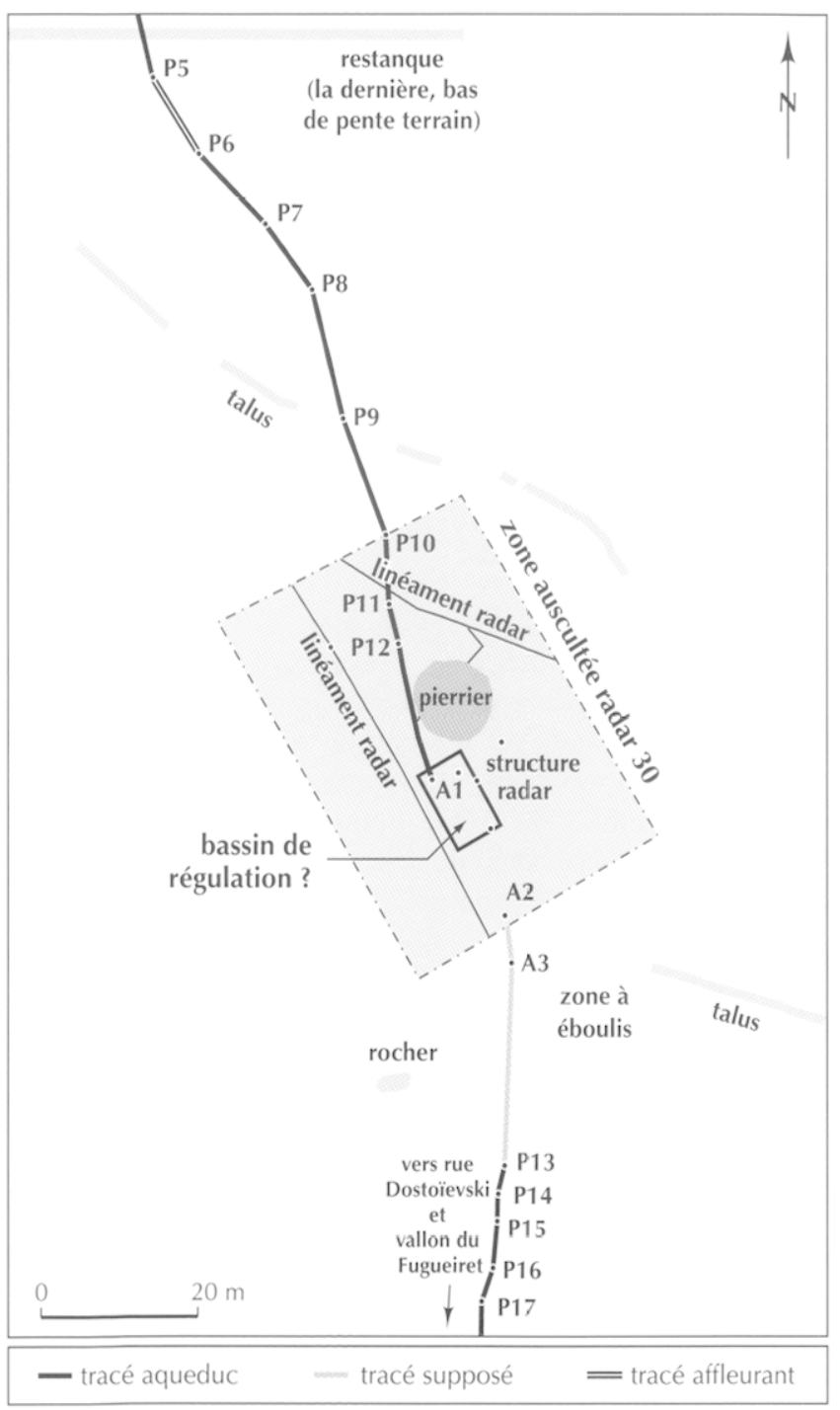

Fig. 8 - Aqueduc d'Antipolis, la descente INPI, canal et structure rectangulaire détectés par radar: bassin de régulation? (I)AO P. Garczynski, CNRS, d'après Telluris).

et les cartes anciennes. Le gain de longueur est de $3350 \mathrm{~m}$. Le fil d'eau du canal se trouve à $12 \mathrm{~m}$ sous le niveau de la route des Lucioles (D 504) qui suit la ligne de crête.

Il s'agit ensuite du problème inverse : éviter le contournement d'un vallon s'évasant en un cirque assez étendu et aux pentes douces, présentant donc un grand périmètre pour une altitude très peu variable. La solution adoptée est un tracé suivant la pente du terrain, descendant directement vers le Fugueiret ; le gain de longueur de canal est ainsi d'environ $2300 \mathrm{~m}$.

Le relevé des altitudes de huit sections du canal réparties sur $83 \mathrm{~m}$ semble montrer deux pentes différentes, adaptées au profil du terrain (fig. 7). Si cette solution entraîne une perte de charge importante, celle-ci est à peu près compensée par le gain issu du choix précédent.

Le terrain a autrefois été aménagé en restanques ; de ce fait, à certains endroits, la voûte se trouve en affleurement. Le sommet du canal est ainsi visible en plusieurs points du fait de cassures, ainsi que par le creusement d'un chenal d'écoulement des eaux de pluie. Les parties intactes et visibles du canal montrent, dès le premier tronçon, une voûte dans son état de construction, sans concrétion en ogive. En bordure du terrain voisin, le canal a été partiellement détruit sur $2 \mathrm{~m}$, ce qui a fait apparaître les concrétions internes importantes formant banquette.

À la base de cette descente, là où le terrain reprend une faible pente et l'aqueduc une configuration normale, on s'attendrait à trouver une installation destinée à ralentir le courant de l'eau dans le canal. Une opportunité exceptionnelle s'est présentée en 2002 par une offre de service à titre expérimental et gracieux de la société Telluris, spécialiste en détection de sous-sol par radar. Ce secteur semblant prédestiné pour une telle recherche, il leur fut confié, et le résultat de leurs travaux fut positif : le canal fut détecté à partir de la zone où il n'était plus repérable en surface ; dans le milieu de cette zone, le tracé est coupé par un rectangle de $6,50 \mathrm{~m}$ par $10 \mathrm{~m}$ qui pourrait être un bassin de régulation (fig. 8).

\section{LE CHANTIER DOSTOÏEVSKI/LEFÈBVRE}

Ici encore ce sont des travaux de réseau qui ont coupé le canal. Ce site, en aval d'un tronçon dans lequel l'eau, fortement agitée, a subi un dégazage important, présente un système de concrétionnement très particulier : lamines sensiblement horizontales et non plus en gobelet, et de nature calcitique très compacte et très dure (voir infra, p. 33). À quelques dizaines de mètres de là et tout près du Fugueiret, le canal sans voûte est visible en affleurement sous quelques centimètres de terre.

l.e franchissement du Fugueiret ne semble pas avoir laissé de trace. 
Fig. 9 - Aqueduc d'Antipolis, vue générale du pont à une arche en élération. On remarque la limite inférieure des concrétions externes qui donnent le profil du talus à l'époque romaine (cliché P. Garcz)nski, (NRS).

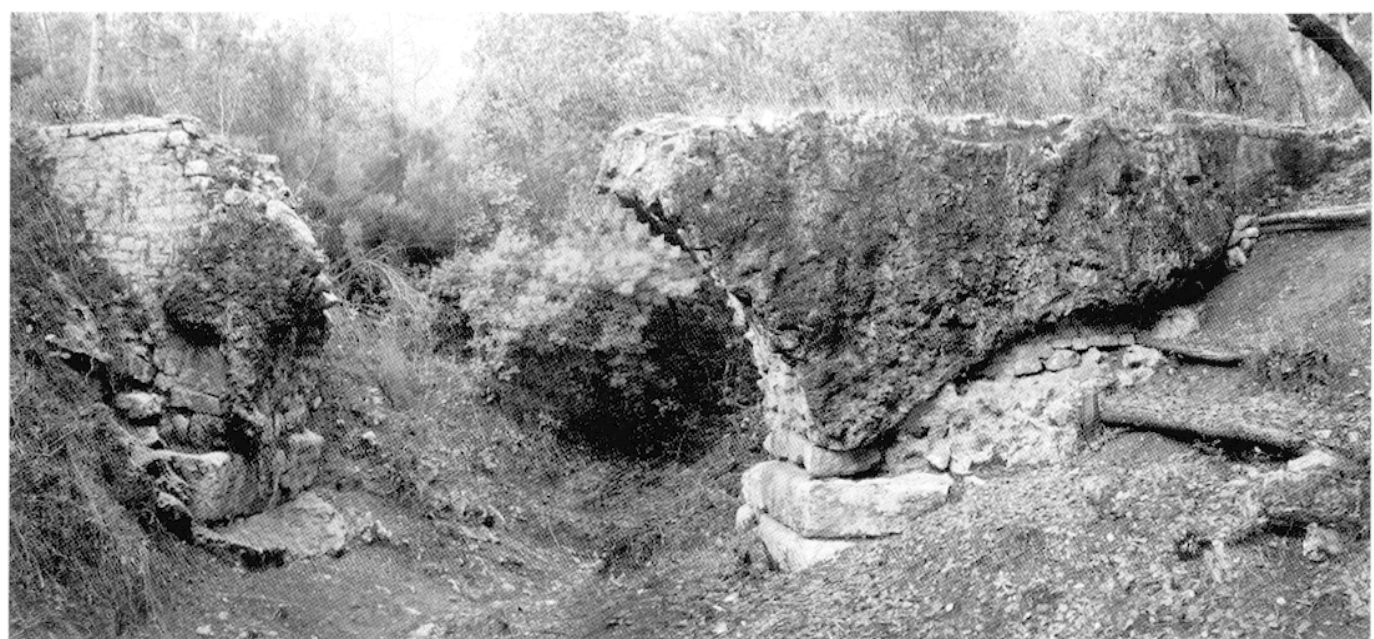

\section{DU FUGUEIRET A LA VALMASQUE}

\section{LA COLLINE DU FUGUEIRET}

La colline porte le nom du cours d'eau mais est aussi bordée par la Valmasque à l'est ; elle est contournéc d'ouest en est par l'aqueduc, sur la rive droite du Fugueiret : en prospection, le canal a été repéré en plusieurs endroits.

\section{LE PONT À UNE ARCHE, LE FUGUEIRET}

Ce pont franchissait un petit affluent intermittent de la Valmasque. Bien qu'effondré, cet ouvrage est important; c'est en effet le seul des trois ponts à présenter sur le tablier des culcés des vestiges en élévation du canal aérien (fig. 9).

Les sondages pratiqués en 2001 et 2002 ont permis de retrouver la même technique observée jusqu'à présent (fig. 10) : un fond de béton moulé, surmonté de deux piédroits de quatre assises de moellons et d'une voûte (fig. 11); cependant, là où le canal sort de terre, alors que les piédroits doivent présenter un parement extérieur, celui-ci est incliné de $37^{\circ}$ par rapport à la verticale, ce qui le fait plus large à la base $(67 \mathrm{~cm})$ et sensiblement de la même largeur que la voûte au sommet $(37 \mathrm{~cm})$. Le fruit est obtenu par la pose inclinée des moellons de parement et non par une taille en biais. Autre particularité apparue : des murets formant coffrage pour le béton moulé, ou contre-parements intérieurs du pont, remplaçant en quelque sorte la tranchée taillée dans la roche lorsque le canal est enterré. Le piédroit avec parement extéricur incliné repose à la fois sur le muret et le béton (fig. 12).

Les concrétions internes forment une masse importante dans le fond du canal et se prolongent par un bloc qui vient sur la culée, en avant des derniers vestiges de piédroits. Les parements des culées sont recouverts d'une épaisse masse de concrétions résultant de fuites importantes, et la limite inférieure de ces concrétions, visibles des deux côtés ct sur les deux culées, donne le profil du talus à l'époque romaine. Les culćes sont fondées sur un massif de maçonnerie débordant, rendu visible par l'érosion du talus, et cette fondation est complétée, au droit des intrados de l'arc, par de gros blocs taillés débordants, installés dans une tranchée de fondation creusée dans le rocher, remarquablement visible sous la culée aval. Le départ de l'arc sur la culée aval, conservé côté ouest sur la hauteur de deux ou trois claveaux, montre la présence d'une archivolte plate (voir infra, p. 25).

\section{LE FUGUEIRET 1}

Au sud, un autre petit vallon à cours d'eau occasionnel est franchi par le canal sans ouvrage particulier: le canal est construit en travers du lit. L'eau passe par-dessus, ce qui l'a en partie détruit, ne laissant qu'une épaisse banquette de concrétions et la partie du béton moulé qui la supporte, mais la maçonnerie est visible immédiatement en aval: seul cas observé pour cet aqueduc, le canal est couvert par des dalles de grandes dimensions. Le site, qui n'a pas encore été étudié, est difficilement accessible et inhospitalier en raison de la végétation.

\section{LE PONT DE LA VALMASQUE}

Peu avant ce pont, le canal est visible le long d'un chemin, du fait de l'effondrement du sommet de la voûte sur $1 \mathrm{~m}$ environ.

Ic pont franchit la Valmasque en deux arches égales. Bien que très dégradé, il est encore debout, conservé et complet, à l'exception du canal lui-même arasé au niveau du tablier. Comme pour le pont à une arche, la végétation est très importante, par comparaison avec des périodes antérieures, comme on peut le constater sur des photos anciennes (fig. 13). Le pont est dans une situation foncière complexe, à la fois sur cinq parcelles appartenant à quatre propriétaires différents, et sur deux communes; il n'a pas encore pu faire l'objet de chantier et de relevé. Afin qu'il puisse être consolidé et protégé, son classement s'avère urgent.

À la sortic du pont se situe le raccordement avec la branche de la Valmasque, sous la chaussée du chemin des Trois-Moulins (CD 35a), ancienne route de Valbonne. 


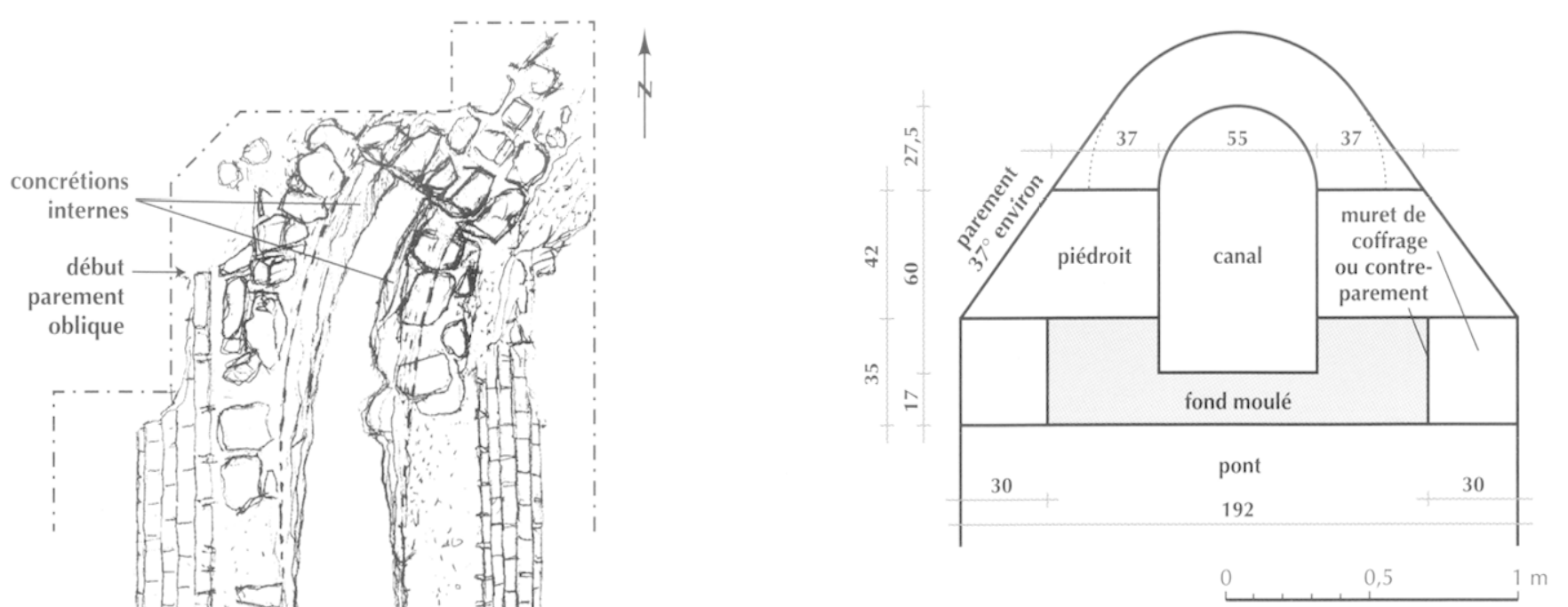

Fig. 11 - Aqueduc d'Antipolis, coupe sur le canal du pont à une arche (DAO P. (jarczynski, CNRS).

repérés et identifiés deux passages à proximité d'un ancien barrage de récupération du cours d'eau (cultures et alimentation de moulins); la connaissance de cette branche en amont est encore insuffisante, de plus la zone est difficilement accessible et nécessiterait un débroussaillage préalable important.

\section{LE SITE ESCOTA 3}

Une section a été mise au jour à quelques mètres en amont de la jonction des deux branches, lors de la construction par Escota ${ }^{20}$ d'un bassin de rétention. La section observée, réduite au fond moulé et à une assise de piédroit, se trouvait dans la berme du trop-plein de ce bassin.

\section{LE CHEMIN DES TROIS-MOULINS}

Ce site a fait l'objet, au cours de l'hiver 2000-2001, d'une opération de sauvetage due à la pose d'une ligne de fibre optique en tranchée le long de la voie qui recouvre le canal, ou ce qu'il en reste (fig. 14). Elle a permis d'observer le canal de l'intérieur sur plusieurs mètres, car la tranchéc s'y est, en quelque sorte, insérée juste avant la zone de sondages (fig. 1.5).

concrétions externes $1 \mathrm{~m}$

Fig. 10 - Aqueduc d'Antipolis, plan du pont à une arche: 1-4, assises des piédroits; a-c, assises du muret de coffrage ou du contre-parement (relevé P. (Garczynski et J. Foucras, (NRS).

\section{LA BRANCHE DE LA VALMASQUE}

Les seuls vestiges étudiés ont fait l'objet des chantiers du site Escota 3 et du chemin des Trois-Moulins, à quelques mètres l'un de l'autre. Dans le vallon et le parc de la Valmasque ont été
Le canal est complet jusqu'à la quatrième assise de piédroit, la voûte ayant été arasée par la construction de la chaussée. Six sondages ont été réalisés tous les $20 \mathrm{~m}$.

Le premier sondage se trouve encore sur cette branche de la Valmasque. Malheureusement le canal a déjà été détruit, en grande partie par une ancienne tranchée de gaz. Le rocher, là aussi, a été entaillé pour permettre la construction du canal ; le lit d'attente de la voûte (piédroit de droite) est constituć d'un lit de mortier soigneusement lissé et légèrement incliné vers l'intérieur, donc déjà rayonnant et qui préfigure les joints de la voûte. Cela n'a pas été observé dans les coupes du canal enterré.

20. Société des autoroutes Estérel-Côte d’Azur. 


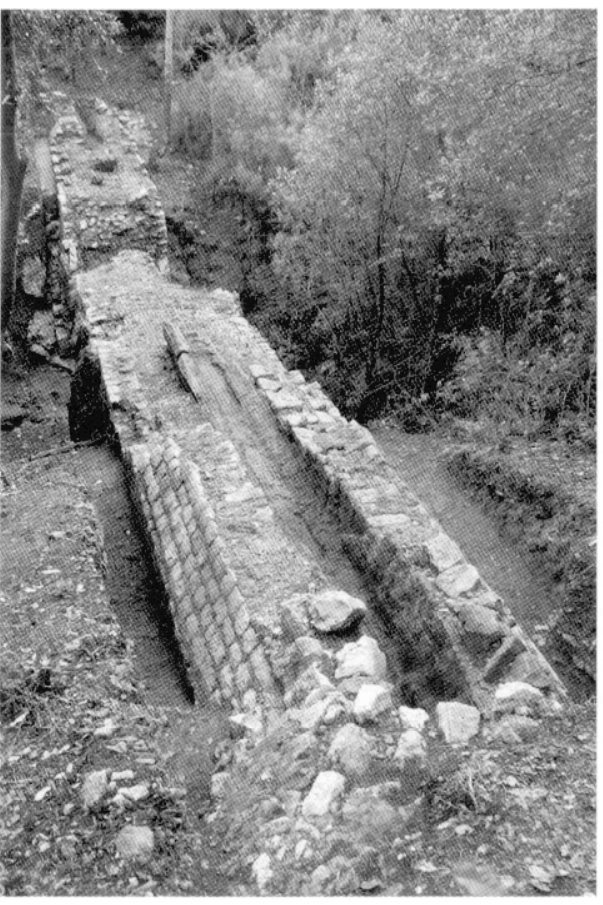

1

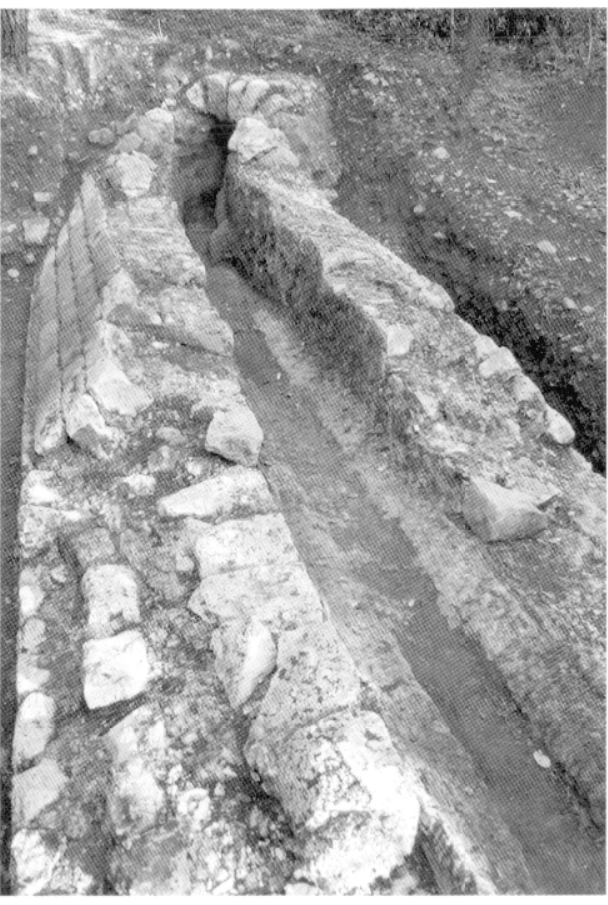

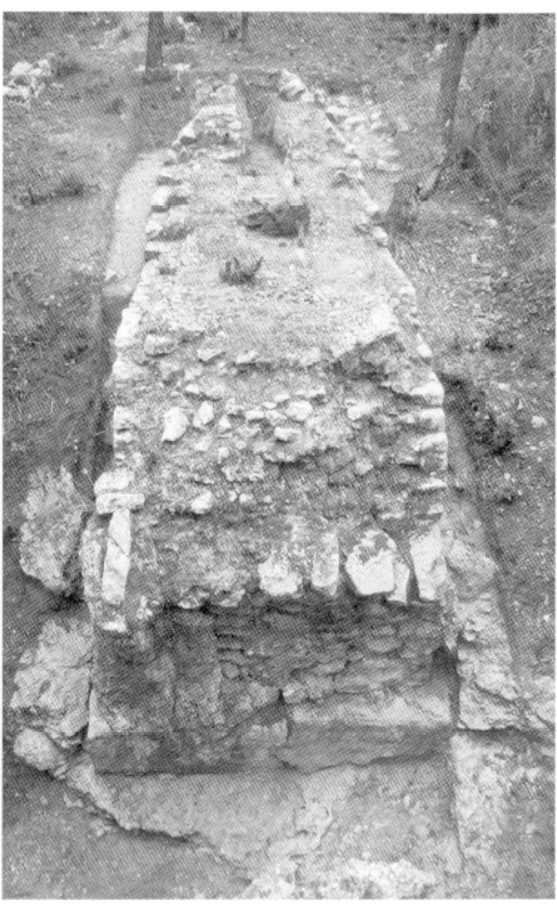

Fig. 12 - Aqueduc d'Antipolis, le pont à une arche : 1, vue générale du dessus, d'amont en aval (au premier plan, le sommet de la voûte du canal dégagé par le sondage); 2, les vestiges du canal sur la culée amont et le canal enterré, dégagé par le sondage (au premier plan à gauche, visible là où le piédroit est détruit, le muret ou contre-parement servant de coffrage au fond moulé); 3, culée aval (on remarque la tranchée de fondation taillée dans le rocher; au sommet du pont à gauche, le muret de coffrage du fond moulé en avant de la partie conservée. du piédroit) (clichés P. Garczynski, CNRS).

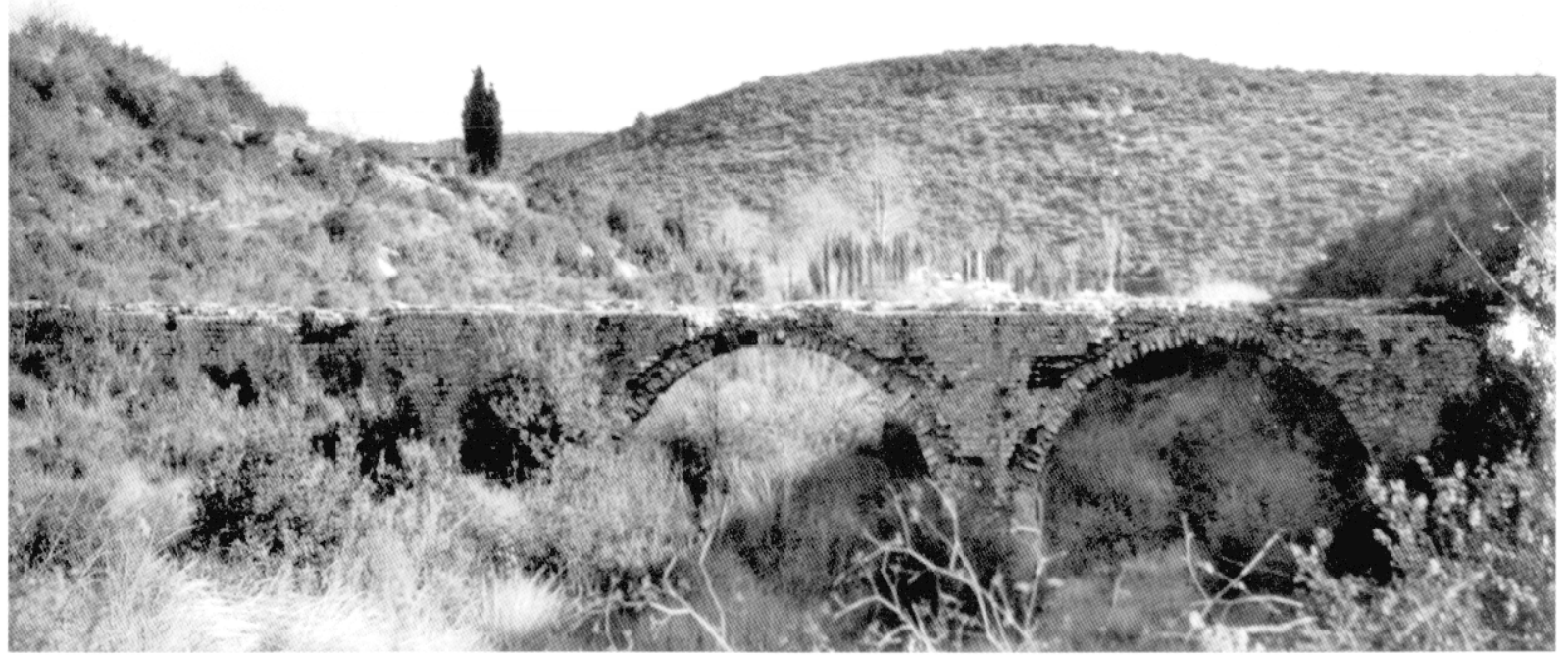

Fig. 13 - Aqueduc d'Antipolis, le pont de la Valmasque, vue générale (cliché M. ('rreenwood, vers 1950). 


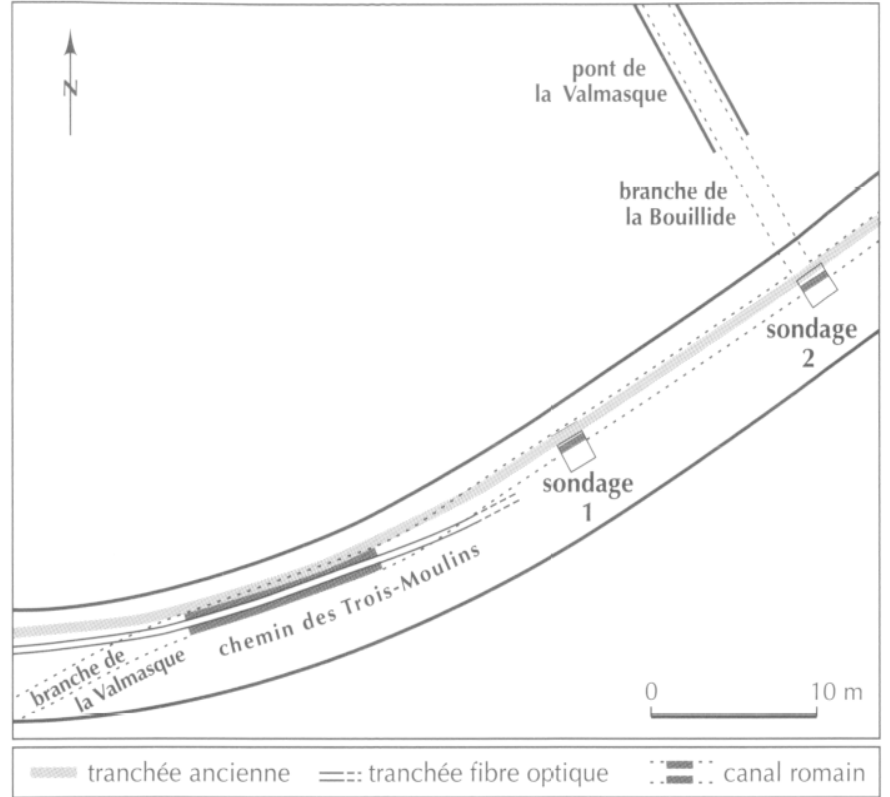

Fig. 14 - Aqueduc d'Antipolis, plan du chantier du chemin des Trois-Moulins (DAO P. Garczynski, CNRS).

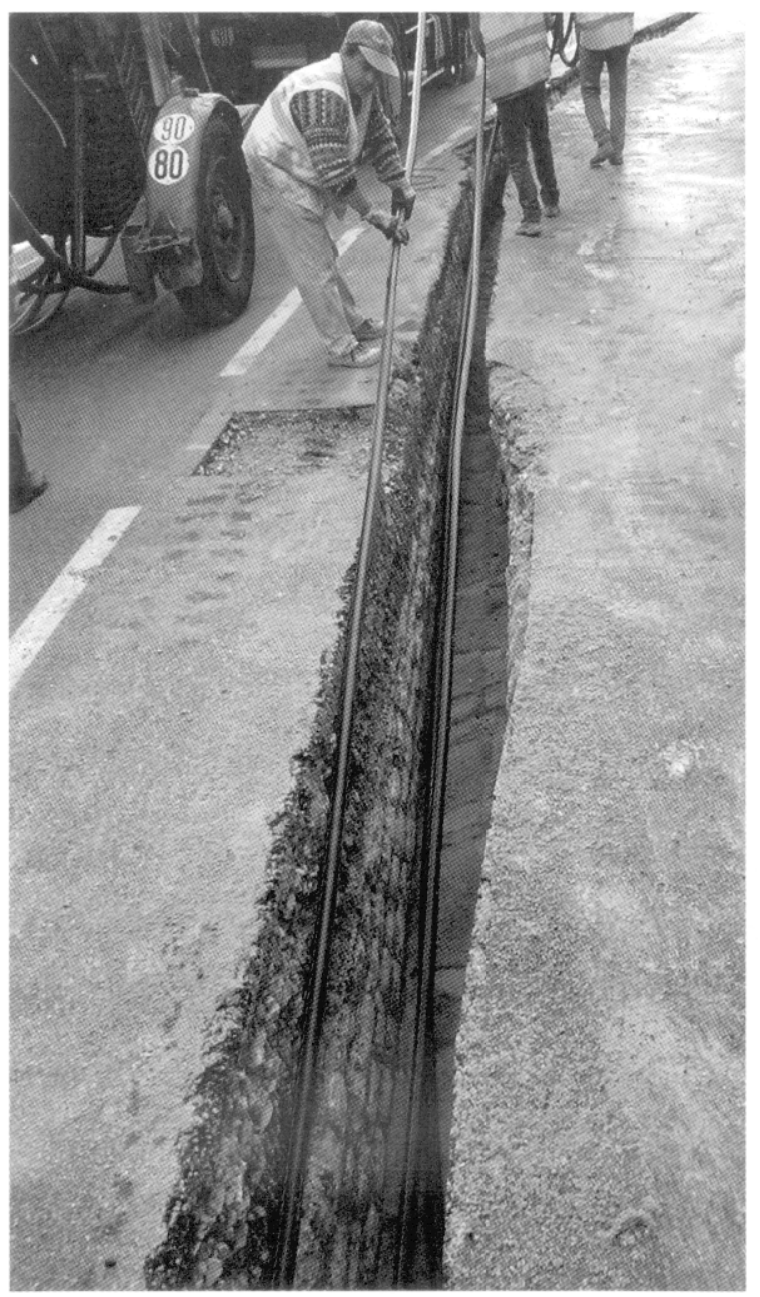

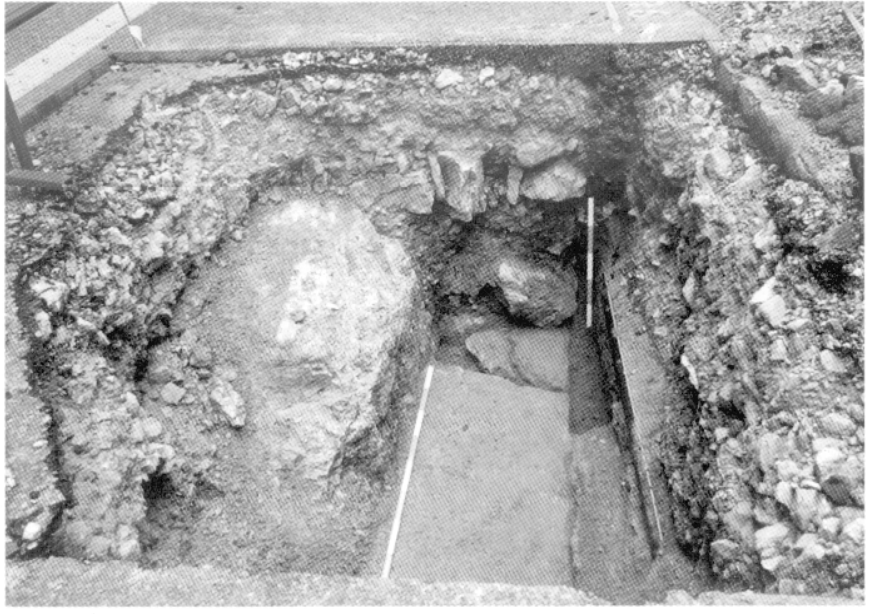

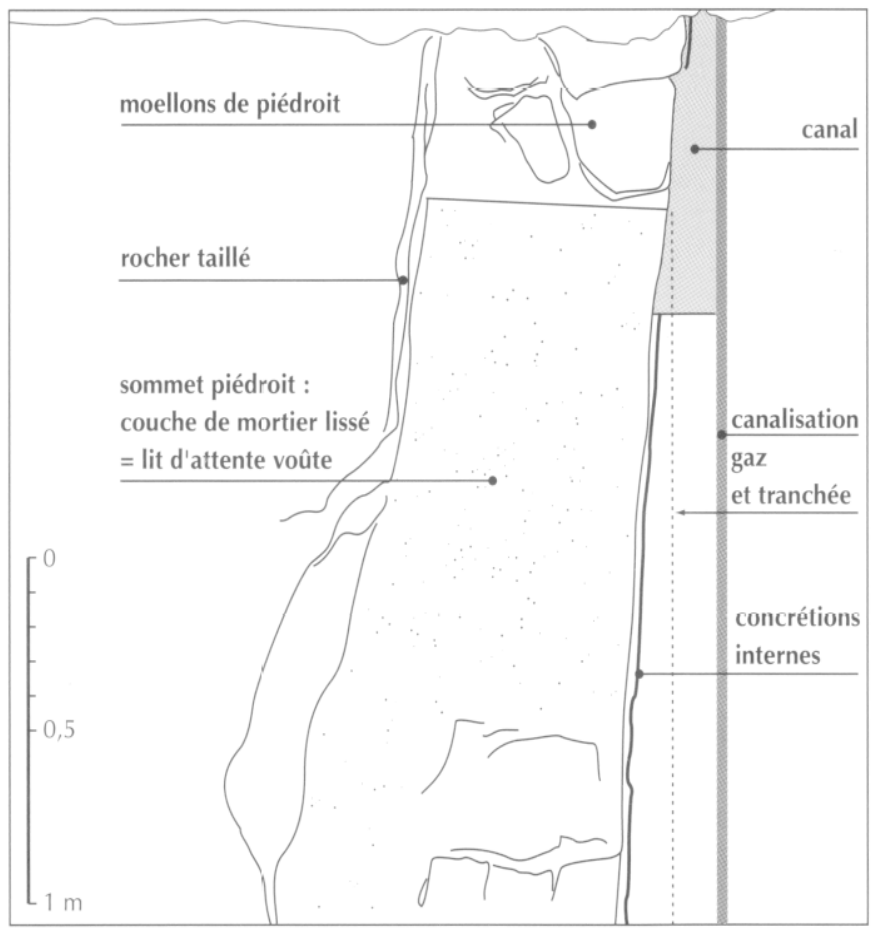

Fig. 16 - Aqueduc d'Antipolis, deuxième sondage au chemin des Trois-Moulins (cliché et plan DAO P. Garczynski et J. Foucras, CNRS). 
Fig. 17 - Aqueduc d'Antipolis, vue d'ensemble vers l'ouest du pont du Goa, de l'aval vers l'amont

(cliché P. Garczynski, CNRS).

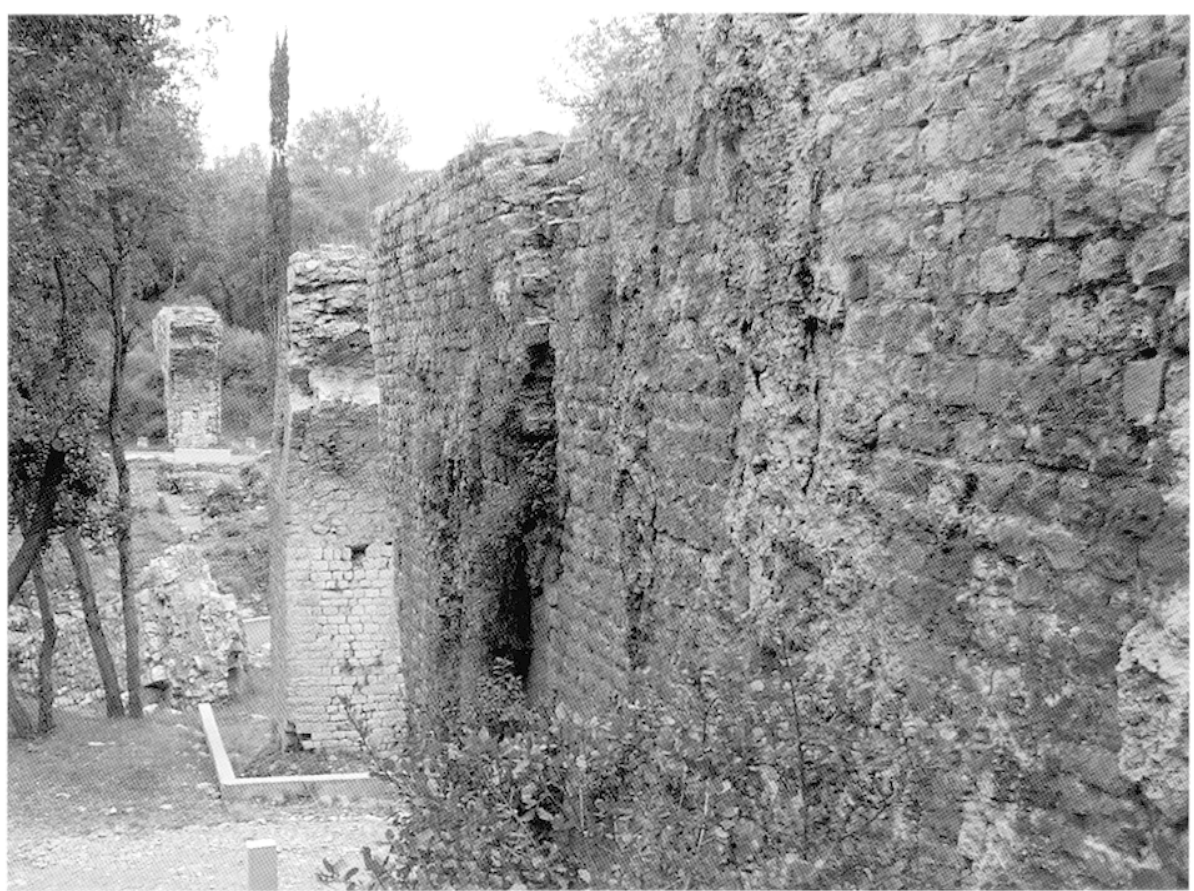

\section{LA BRANCHE COMMUNE}

\section{LE CHEMIN DES TROIS-MOULINS (SUITE)}

Le second sondage a été implanté exactement à la sortie du pont de la Valmasque, au droit de la jonction supposée des deux branches, afin de mettre au jour le raccordement des deux canaux. Malheureusement, là encore, la tranchée ancienne a définitivement détruit le piédroit gauche qui aurait pu montrer ce raccordement, ne laissant que le piédroit opposé et quelques centimètres de conduit ; le sommet du piédroit est analogue à celui du premier sondage, lissé et incliné vers l'intérieur (fig. 16). Ce détail ayant été déjà observé dans le premier sondage conforte l'idée de l'antériorité de la branche de la Valmasque par rapport à la branche de la Bouillide, ou du moins de leur contemporainéité. Cependant, un indice infime - un fragment de mortier en coupe avec une couche d'enduit verticale, au-dessus du niveau de la voûte et en prolongement du piédroit - indique l'absence de voûte à cet endroit, et la possibilité d'une installation particulière liée à la rencontre des deux canaux : chambre ou regard?

\section{LE SITE DU GOA}

Ce pont qui enjambe le Goa est malheureusement très dégradé. Il ne reste en situation que la culée amont, la base de la pile I, la pile IV et la culée aval (fig. 17). Cela a d'ailleurs permis de nombrcuses spéculations, en particulier sur le nombre des arches qui a été estimé de trois à sept suivant les auteurs, le nombre le plus courant étant de quatre. Nous pouvons cependant conclure d'après nos observations qu'il s'agit d'un pont à cinq arches, ce qui en fait l'ouvrage le plus important de l'aqueduc.

Le site a fait l'objet d'une étude approfondie à l'occasion du réaménagement de la gare autoroutière d'Antibes et de la construction d'un nouveau péage, sur un terrain acquis par la société d'autoroute qui s'engagea à le réhabiliter et à l'aménager. C'est ainsi qu'apparurent des vestiges auparavant invisibles par l'absence d'entretien du secteur.

L.e Goa a dû être autrefois un grand cours d'eau, du moins à l'époque romaine si l'on en juge par la largeur du vallon. Actuellement, bien qu'ayant un petit débit, on a pu remarquer à l'occasion de pluies torrentielles qu'il peut grossir considérablement en quelques minutes, ce qui est un facteur de dispersion des petits blocs effondrés, et de l'enfoncement progressif des plus gros. Le site se présente donc avec des éléments en situation, mais aussi avec des blocs effondrés apparus grâce à la réhabilitation de cette zone. Quatre sondages ont été réalisés : en amont, à l'emplacement de la pilc II, à l'emplacement présumé de la pile III et en aval du pont.

\section{LE SONDAGE AMONT}

Comme déjà mentionné, le canal bâti sur le tablier du pont a complètement disparu. Pour pouvoir relever le niveau NGF du fil d'eau à la mise en cau le plus près possible de l'entrée du pont, mais aussi pour observer la technique de maçonnerie du canal au moment où il passe en surface, un sondage a été pratiqué avant le raccordement avec la culée, à $16 \mathrm{~m}$ du début de la culée amont; il a ensuite été étendu jusqu’à la \%one recouverte par la chaussée du chemin des Trois-Moulins (fig. 18).

Au plus près de la culée, le canal dégagé est conservé jusqu'à la deuxième assise de moellons du piédroit ; à mesure que nous avons étendu le sondage vers l'ouest, le canal étant plus enfoncé, ont été dégagés les deuxième et troisième assises, puis les premiers claveaux de la voûte. La particularité apparue par ce sondage est, comme au pont à une arche, un parement extérieur du canal incliné à $37^{\circ}$, qui commence là 


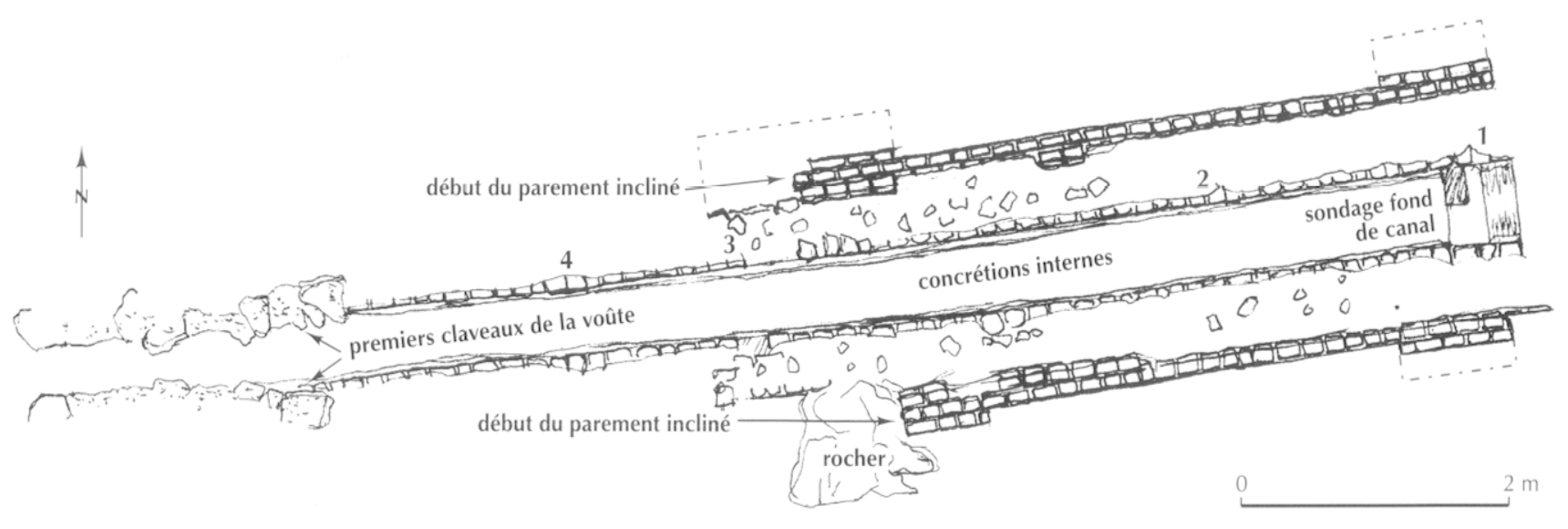

Fig. 18 - Aqueduc d'Antipolis, plan du sondage amont du pont du Goa : 1-4, assises de piédroits (relevé P. Garczynski et J. Foucras, CNRS).

où le canal sort de terre et doit avoir des faces extérieures construites (fig. 19).

\section{LE PONT}

\section{Les vestiges en élévation}

Ces vestiges se composent de la culée amont, de la base de la pile I dégagée par sondage, de la pile IV et de la culée aval (fig. 20).

La culée amont est constituée de deux parties rectilignes faisant entre elles un angle de $164^{\circ}$ sans arc de raccordement. Le canal qu'elle supportait a été arasé au niveau du radier. Une partie importante de la première arche est conservée jusqu'au premier tiers environ (fig. 21). L'arche, comme les autres encore observables partiellement, est formée de deux arcs de tête constitués par de grands claveaux rayonnants, taillés. Entre les arcs de tête, comme pour la voûte du canal, ce sont des pierres utilisées au mieux de leur forme allongée qui constituent des simili-claveaux et dont la cohésion est assurée par le mortier de chaux. Un détail architectural tout à fait particulier peut être observé : il s'agit d'un bandeau de moellons, à plat sur l'extrados de l'arc et soulignant ce dernier, que nous appelons " archivolte plate ", puisque le terme archivolte désigne une moulure. Son rôle est esthétique et, dans le cas des trois ponts, cela la rend plus originale pour des monuments somme toute de facture assez fruste.

Le dégagement de la base de la pile I n'a pas apporté d'information particulière, sinon de donner précisément la dimension de la première arche $(5,30 \mathrm{~m})$ et de montrer que, comme la pile IV, elle n'était pas contrefortée. Elle comporte une dizaine d'assises conservées, le sommet se trouvant à quelques centimètres sous terre. Les premières assises, parmi lesquelles le parement, qui est constitué de moellons plus grands, sont posées directement sur le rocher et il n'y a pas d'élément de fondation.

Des traces de mortier sur la roche, dans le lit du Goa, sont apparues à la suite des travaux d'engins mécaniques destinés à casser le rocher pour déplacer légèrement le cours d'eau et l'éloigner des vestiges au sol; elles se sont avérées correspondre à l'emplacement de la pile II telle que la restitution la situe ; en

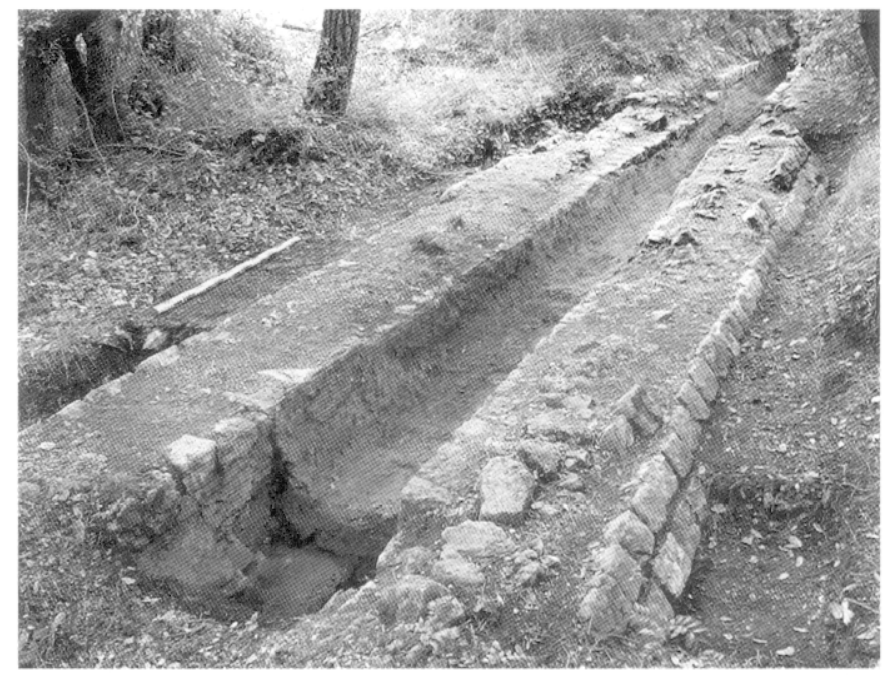

Fig. 19 - Aqueduc d'Antipolis, le sondage amont du pont du Goa, vue vers l'ouest (cliché P. Garczynski, CNRS).

outre, le profil du rocher, particulier à cet endroit, permet de penser qu'il a été taillé pour permettre l'assise de la pile.

Un sondage fait à l'emplacement présumé de la pile III n'a pas permis de découvrir une maçonnerie organisée, mais un massif de pierres et de mortier appuyé d'un côté contre une face verticale de rocher, qui semble bien correspondre à un blocage destiné à recevoir la pile.

La pile IV comporte de chaque côté les départs des quatrième et cinquième arches (fig. 22); les deux ayant le même plan de naissance, les arches sont donc en principe égales à quelques centimètres près. Cette pile qui s'élève jusqu'au niveau du radier du canal est en place, mais elle a pris une légère inclinaison vers le nord-ouest.

La culée aval est incomplète au niveau du piédroit de la cinquième arche qui n'est plus visible; un rapide sondage au pied de cette partie détruite a permis de retrouver les premières assises et la verticale du piédroit, et de donner ainsi la largeur précise de l'arche, $5,30 \mathrm{~m}$, égale à celle de la 


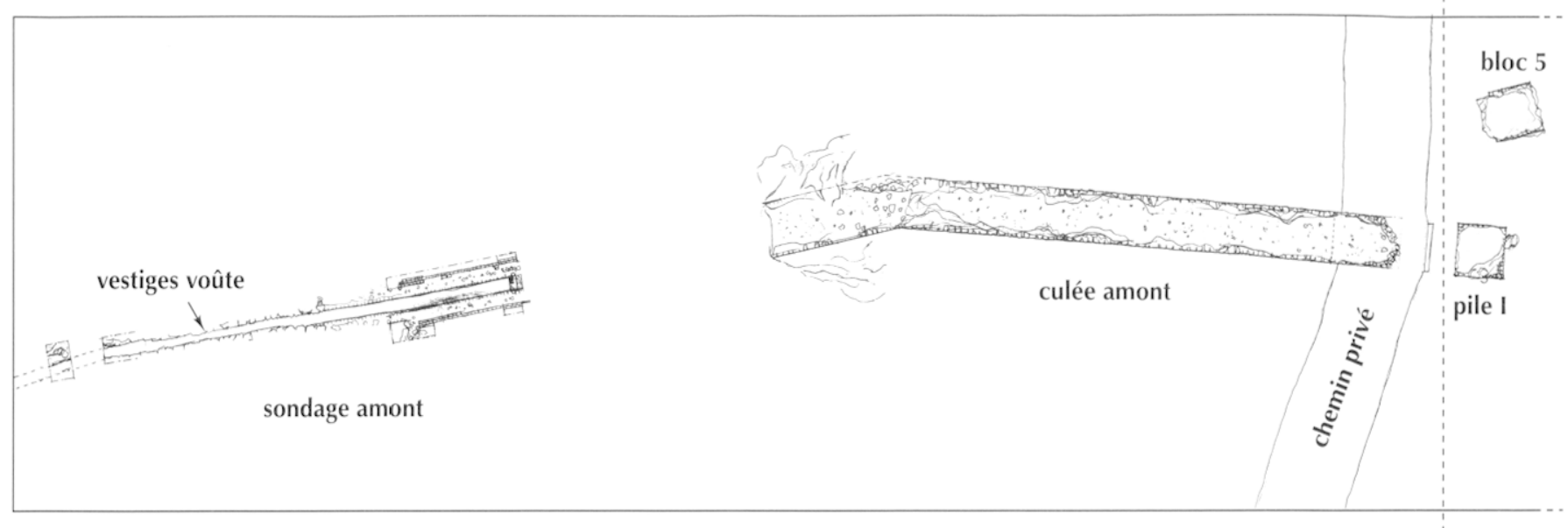

Fig. 20 - Aqueduc d'Antipolis, plan du pont du Goa (relevé P. Garczynski et J. Foucras, CNRS).

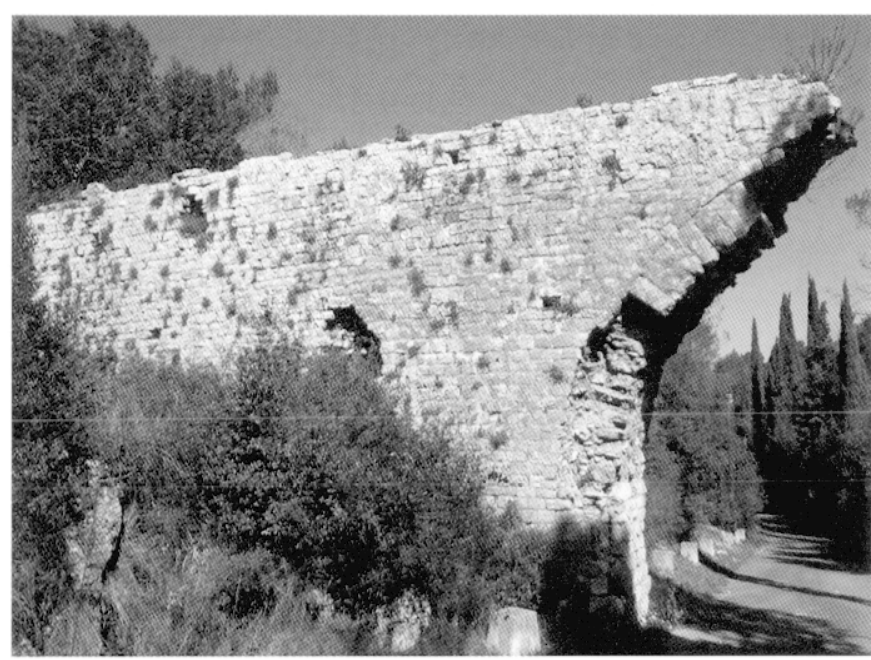

Fig. 21 - Aqueduc d'Antipolis, la culée amont du pont du Goa (cliché P. Garczynski, CNRS).

première. Une carte postale ancienne, datée de 1910 grâce à son timbre, prouve que cette destruction est récente: on distingue sur la photo une partie de la retombée de l'arche sur ce piédroit. Cette destruction moderne permet d'observer le blocage intérieur de la maçonnerie, en particulier des lignes de séparation des banchées ayant servi à la mise en place de ce blocage, par couches successives de 40 à $70 \mathrm{~cm}$.

La construction est fendue du haut en bas par une large fissure ; la partie côté piédroit, qui se trouve ainsi séparée du reste, accuse une légère inclinaison vers le sud. Nous reviendrons sur ces inclinaisons opposées (pile IV et culée) à propos des causes possibles de la destruction de l'ouvage. Les deux faces sont couvertes d’importants dépôts de concrétion, témoins de défauts d'étanchéité du canal.

\section{Les vestiges effondrés}

Ils sont au nombre de cinq, mais seuls quatre d'entre cux ont présenté un intérêt et ont été étudiés.
Le bloc 1 serait la tête de la pile III, il comporte des parties des troisième et quatrième arches, lesquelles apparaissent nettement inégales (fig. 23) ; la plus petite, qui correspondrait à la quatrième arche, présente un intrados et des claveaux très bien conservés. Avec l'inégalité des arches, l'information importante donnée par ce bloc comme par le suivant est la présence de contreforts sur les deux faces. Ce qu'il en reste montre qu'ils avaient un fruit important, donc une grande épaisseur à la base, ce qui rappelle encore l'aqueduc de Fréjus : de $2 \mathrm{~m}$ à 2,50 $\mathrm{m}$ de large, alors qu'elle est de $0,20 \mathrm{~m}$ ou nulle en haut, au niveau du tablier. La seule observation des blocs 1 et 2 a permis de comprendre que ce pont avait une arche centrale plus grande, encadrée et soutenue par deux piles renforcées par des contreforts, enfin que l'on pouvait proposer une restitution à cinq arches.

Le bloc 2 serait alors la tête de la pile II. Comme le précédent, il comporte des parties des deuxième et troisième arches inégales, ainsi qu'un contrefort sur chaque face. Il est moins bien conservé : les arcs de tête notamment ont disparu.

Le bloc 3, sensiblement parallélépipédique, ne comporte pas de face caractéristique autre que des parements verticaux ct le radier du pont; il serait donc un élément de maçonnerie situé au-dessus de la pile I.

Le bloc 5 est très enfoncé et en grande partie sous le bloc 2 dont il s'est détaché; il est intéressant car il comporte les départs des deuxième et troisième arches sur des faces opposées.

\section{La restitution du pont}

Les emplacements des piles II et III ne sont pas précisément connus: les diamètres des trois arches centrales sont donc inconnus. I es deux extrêmes étant égales, tout comme les piles, il est alors probable que le pont était symétrique, et que les deuxième et quatrième arches encadrant l'arche centrale ćtaient aussi égales (fig. 24). La difficulté fut de déterminer le rayon des fragments d'arcs disponibles sur le terrain, étant donné leur état de conservation ou leur faible longueur. Différents systèmes mis en place sur le bloc 1 (ficelles rayonnantes donnant le centre, projection des points sur une règle, 


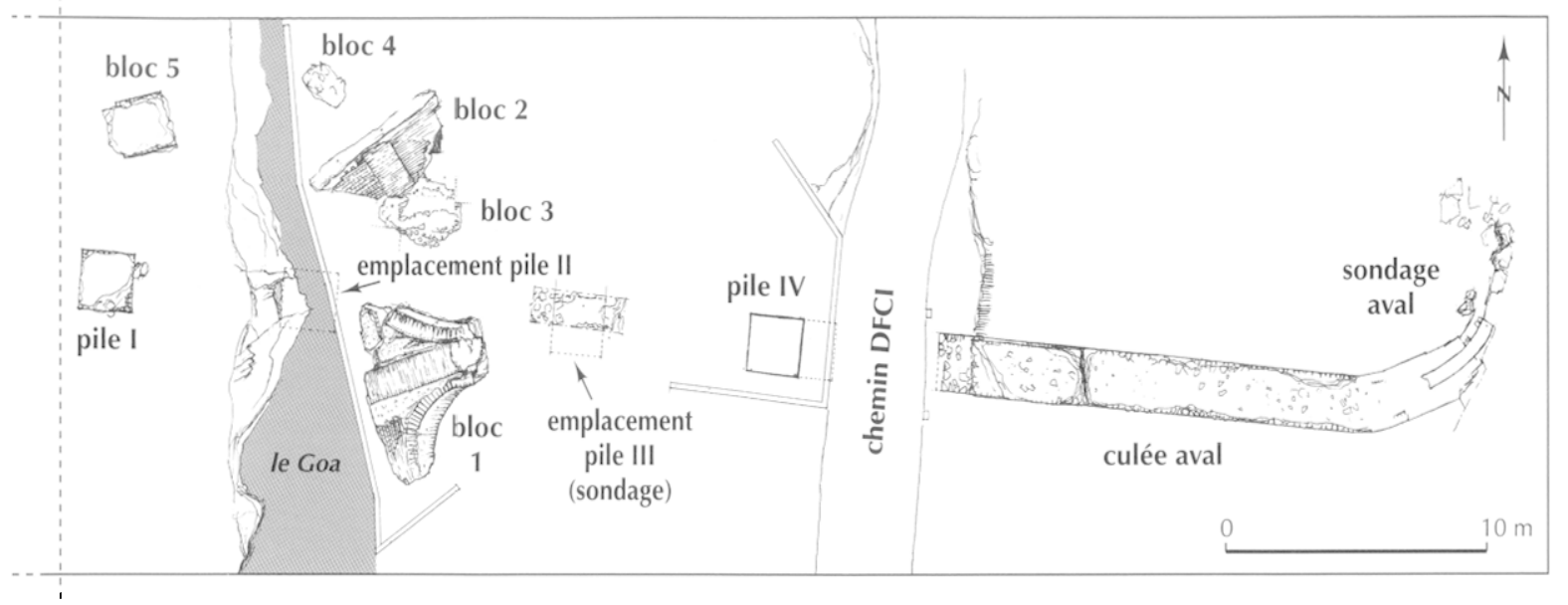

Fig. 20 suite - Aqueduc d'Antipolis, plan du pont du Goa (relevé P. Garczynski et J. Foucras, CNRS).

restitution dans l'espace du plan de naissance, etc.) donnèrent des premières mesures. L'exploitation du relevé topographique et informatique en donna d'autres ${ }^{21}$. Les deux ou trois valeurs obtenues permettent de déterminer une moyenne possible pour le rayon de chacune de ces trois arches : 2,90 m, 4,20 m et $2,90 \mathrm{~m}$, soit respectivement 10,14 et 10 pieds romains (fig. 25 et tabl. I).

Donner une largeur de $5,80 \mathrm{~m}$ (20 pieds) à la quatrième arche implique que son sommet dépasse celui de la cinquième de $25 \mathrm{~cm}$, mais il semble bien que cette hauteur entre sommet d'arc et radier soit différente pour chaque cas ; nous avons fait le choix de l'hypothèse du pont symétrique pour les largeurs d'ouverture. On a d'ailleurs observé certains défauts ou maladresses de stéréotomie. Par exemple sur la face nord de la pile IV, la courbe est aplatie au départ de l'arc et ne correspond manifestement pas au diamètre ; si l'on devait restituer l'arche avec ce seul morceau, le résultat serait erroné. Rien n'empêche de penser que les arches sont légèrement déformées au sommet afin d'obtenir une hauteur de maçonnerie suffisante jusqu'au radier.

La pente du radier n'a pas été prise en compte car elle n'est que de $0,184 \%$, moins de $1,5 \mathrm{~cm}$ pour $7,65 \mathrm{~m}$ : sur la longueur totale du pont $(39 \mathrm{~m})$, la différence de niveau mesurce est de $7,2 \mathrm{~cm}$. Entre les plans de naissance des première et cinquième arches la différence de niveau est de $28 \mathrm{~cm}$, mais elle a pu être moindre car cette pile penche et a pu s'enfoncer légèrement.

\section{LE SONDAGE AVAL}

À la sortie du pont, le terrain remonte beaucoup plus rapidement qu'en amont. Il s'agit du versant de la colline des Croûtons ; le canal apparaît vite, d'abord le béton moulé puis

21. Nous remercions nos collègues de l'Institut de recherche sur l'architecture antique J.-M. I abarthe et I). Téodorescu pour le redresscment en gémétral. les piédroits, tournant pour reprendre une trajectoire de niveau sur le versant. Le sondage n'a pu être pratiqué jusqu'à ce que le canal apparaisse complet car des blocs de rocher effondrés l'en empêchaient.

On retrouve sur la culée les premières assises des contreparements entre lesquels était coulé le fond du canal. Ils n'ont pas été observés en amont car le sondage n'a pas atteint leur niveau en profondeur.

\section{LA COLLINE DES CROÛTONS}

\section{LE CONTOURNEMENT DE LA COLLINE DES CROÛTONS}

Cinq passages de l'aqueduc ont pu être reconnus et relevés (Croûtons 1 à 4 et Cistes) : empierrement de sommet de piédroit en travers d'un chemin, coupe du canal avec concrétions internes en bordure d'une ancienne carrière d'argile, voûte ou partie de voûte visible dans deux petites tranchées naturelles d'écoulement des eaux de ruissellement, enfin sommet de voûte dans une rigole ceinturant l'immeuble le plus au nord d'un ensemble de constructions rue des Cistes, juste avant la destruction du canal par cet immeuble. Ce point marque la fin de la partie relativement bien conservée de l'ouvrage, son premier tiers, puisqu'on pénètre à présent dans la zone urbanisée d'Antibes, et ce jusqu'à la côte.

\section{LE BASSIN SUPPOSÉ DE DÉCANTATION}

Ce bassin, détruit en 1976 pour la construction d'un parking, nous est connu par de brefs articles arec figure de J. Marguier et de M. Compan (fig. 26). Marguier l'authentifie comme romain et pense qu'il s'agit d'un bassin de décantation ou d'une " piscine épuratoire ", mais il aurait pu s'agir d'un bassin de régulation; il semble qu'aucun relevé détaillé mettant en évidence son principe de fonctionnement n'ait été effectué, et les croquis mentionnés indiquent des cotes différentes. 


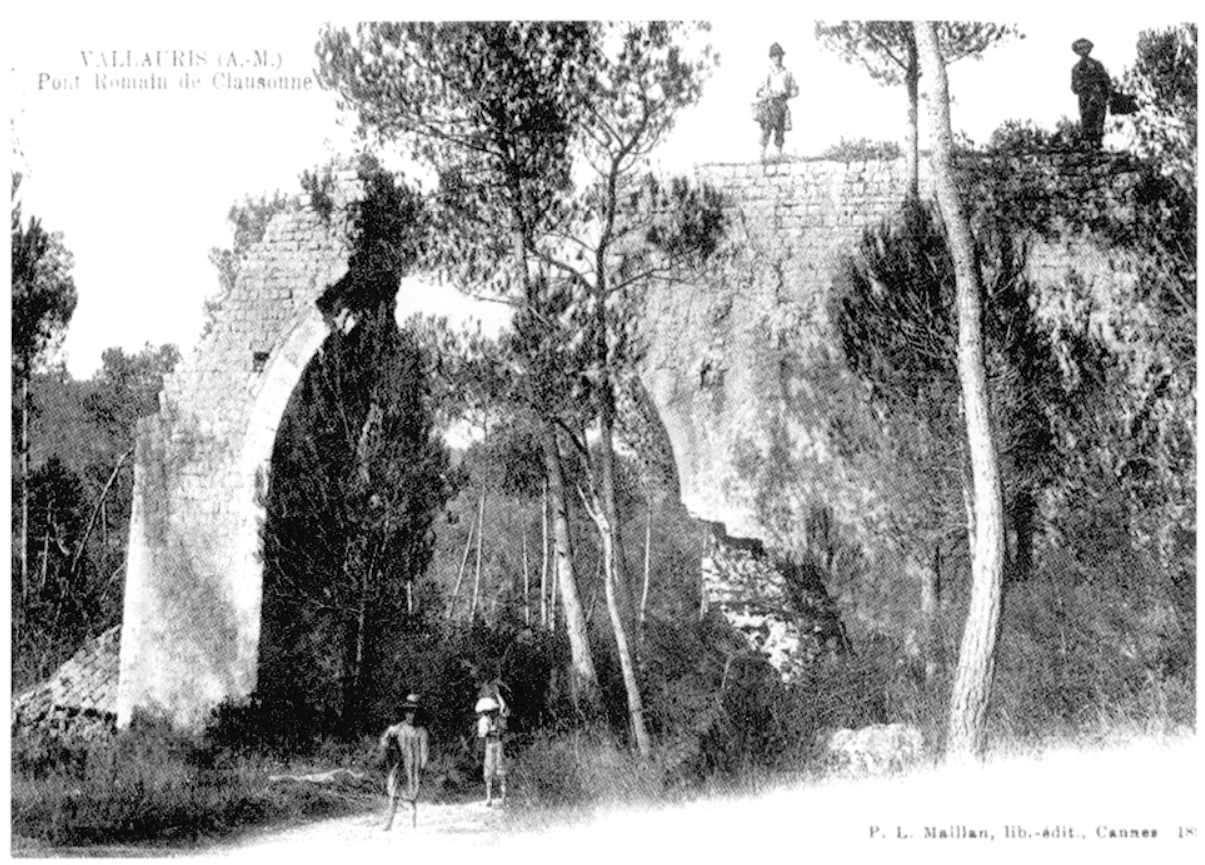

1

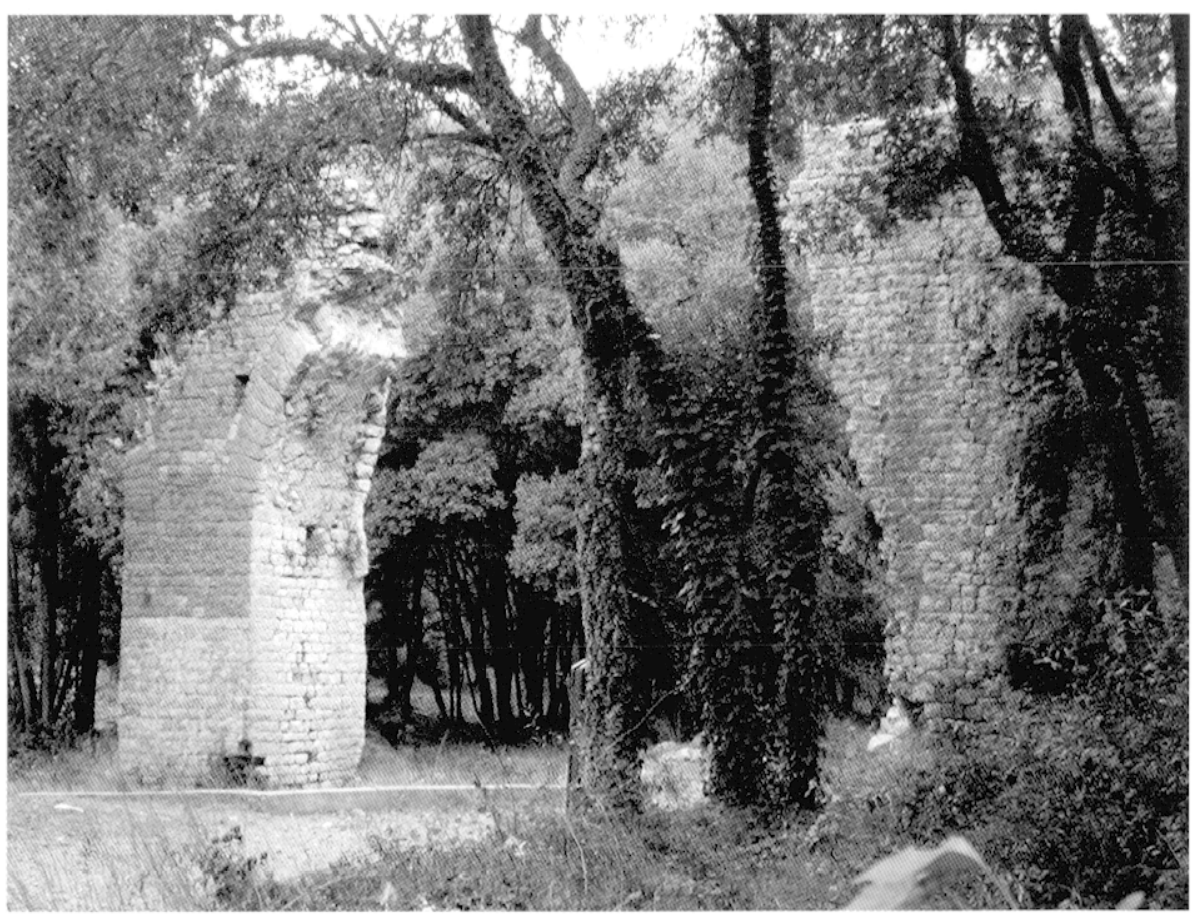

2

Fig. 22 - Aqueduc d'Antipolis, la pile IV et la culée aval du pont du Goa: la photo $n^{\circ} 1$ est une carte postale datée de 1910 sur laquelle on distingue la retombée de l'arc sur le piédroit, côté aval, qui est conservé; ce n'est pas le cas sur la photo $n^{\circ} 2$, une grande partie de maşonnerie s'est effondrée depuis ( $n^{\circ} 2$, cliché P. Garcz)nski, (NNRS).

\section{LES SITES ESCOTA 1 ET 2}

Toujours dans le cadre du réaménagement du péage d'Antibes, une des premières sections apparues (Escota 1) le fut dans la berme d'une grande tranchée destinée à recevoir une buse d'évacuation, à l'intérieur de la boucle de sortie de l'autoroute en provenance de Nice. Quelques dizaines de mètres en amont, la construction d'un grand bassin de déshuilage a fait l'objet d'une surveillance spéciale puisqu'il se trouvait sur notre tracé restitué (Escota 2). Deux sections opposées apparurent effectivement une fois le creusement terminé, très proche du fond de l'excavation.

\section{LE VALLON DU LAVAL}

\section{LE CHEMIN DE SAINT-CLAUdE ET LE CIMETIÈRE}

Le long du chemin de Saint-Claude, des vestiges maintenant disparus ont été vus autrefois à la hauteur de la chapelle. Un peu plus loin, le sommet de la voûte se voyait en travers du 

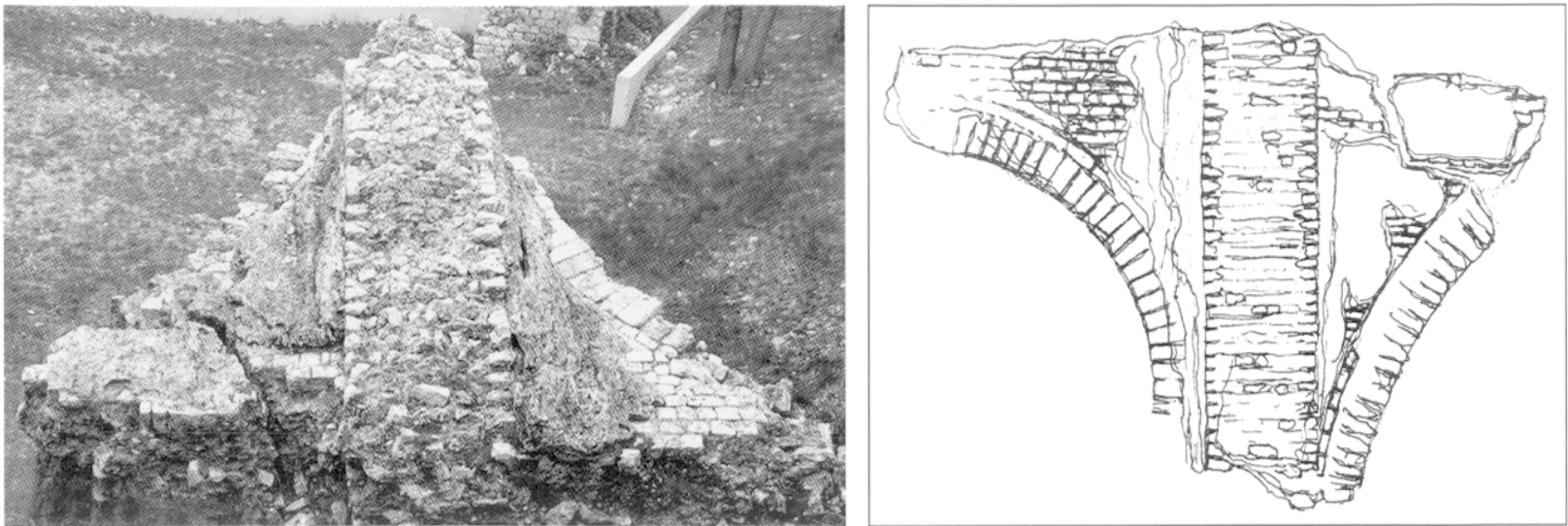

Fig. 23 - Aqueduc d'Antipolis, le bloc 1 du pont du Goa (cliché et relevé P. Garczynski et J. Foucras, CNRS).

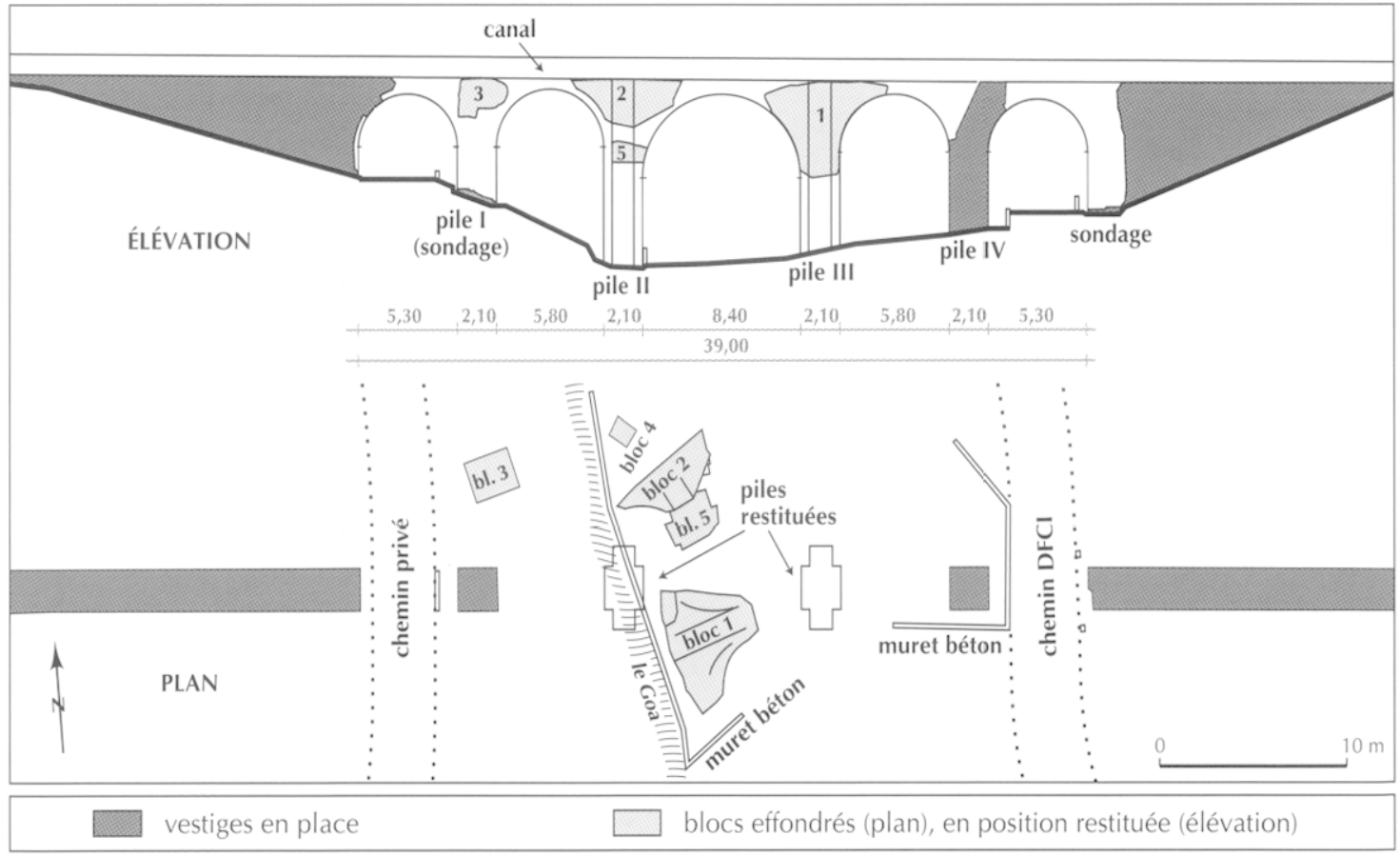

Fig. 24 - Aqueduc d'Antipolis, plan et élévation restituée du pont du Goa (DAO P. Garczynski et J. Foucras, CNRS).

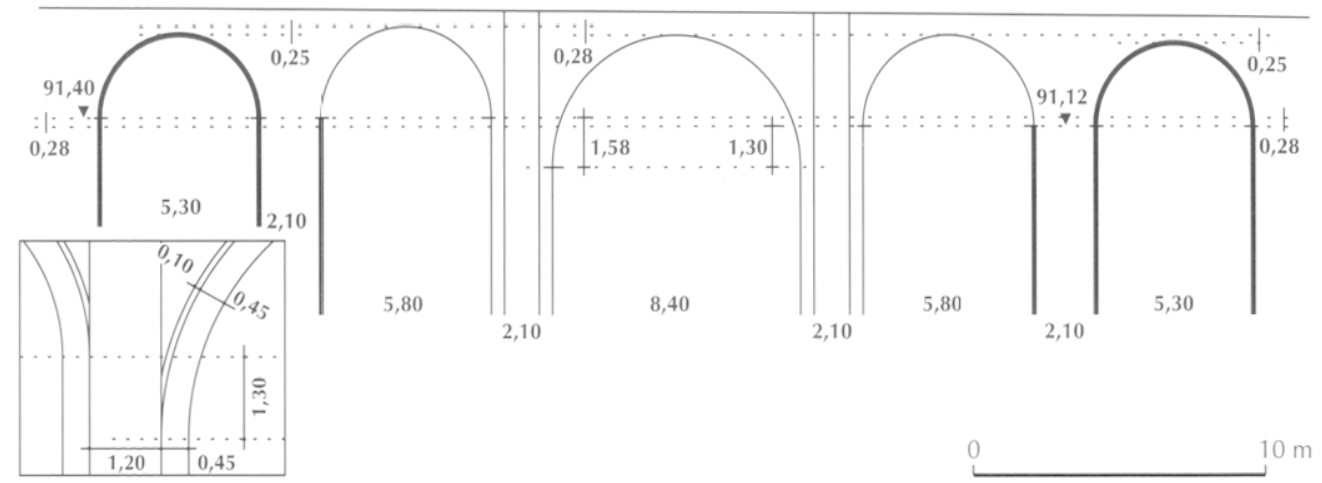

Fig. 25 - Aqueduc d'Antipolis, le pont du Goa : tracé directeur de la restitution, détail des départs de l'arc et de l'archivolte pour les piles contrefortées (DAO P. Garczinski, CNRS). 
Tabl. I - Aqueduc d'Antipolis : mesures des largeurs des 2", 3" et $4^{\prime}$ arches du pont du Goa.

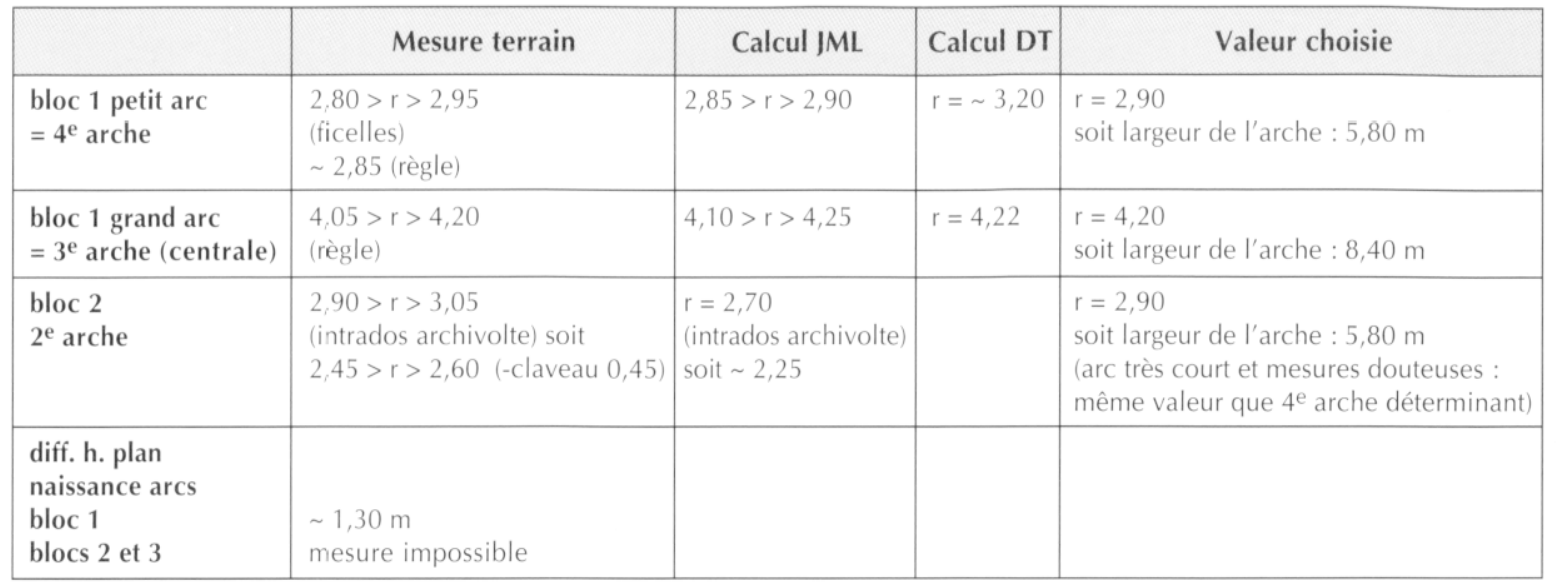
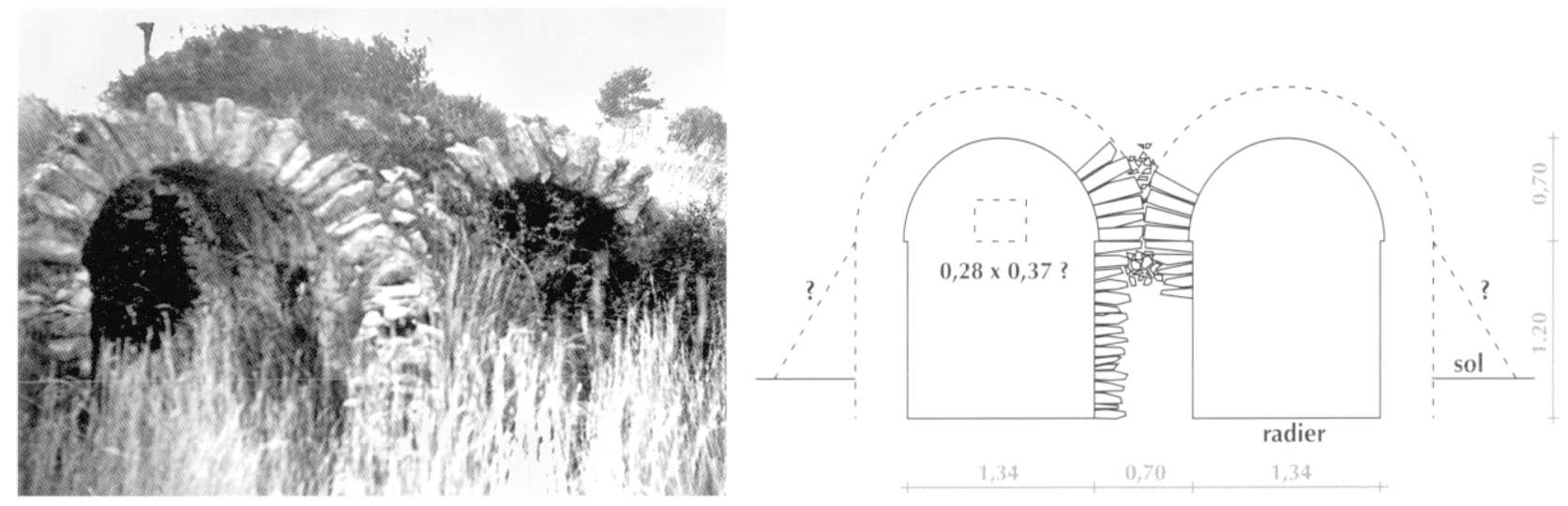

Fig. 26 - Aqueduc d'Antipolis, les Croûtons : bassin supposé de décantation aujourd'hui détruit, rliché ancien et élévation restituée d'après deux dessins sommaires (cliché J. Marguier; DAO P. ('arczynski, CNRS).

trottoir selon le témoignage de notre regretté collègue George Rogers; il avait également mentionné une section visible dans le talus de la rue à la sortie du pont sous l'autoroute. Plus loin selon P. Cosson, l'aqueduc suivait le chemin au sud, puis au nord, et le recoupait à nouveau à hauteur du chemin de Beauvert ; un tronçon de $100 \mathrm{~m}$ était visible le long du Laval. L'aqueduc passait ensuite au milieu de l'ancien cimetière.

\section{LE CHEMIN DU PUY, LA PROPRIÉTÉ HUGUES ET LA RÉSIDENCE DES COMORES}

Deux vestiges sont encore visibles dans ce secteur; l'un connu, dans le jardin d'une propriété privée où seul le haut de la voûte apparaît ; lautre à $5 \mathbf{m}$ de haut dans une paroi de rocher taillée pour les besoins de la construction d'une résidence (fig. 27). Ln grillage de protection recouvrait le tout, envahi par une végétation rendant la coupe à peine visible, et en tout cas inaccessible. Fin 2002, une occasion de l'observer de près s'est présentée lors de travaux de nettoyage et de consoli- dation de la paroi rocheuse, après démontage du grillage. Avec le concours des pompiers d'Antibes et grâce à une grande échelle, photos et mesures ont pu être prises après un nettoyage sommaire. Cette coupe est d'un grand intérêt car elle montre le canal enterré complet, et c'est sans doute la dernière que l'on puisse encore voir. Deux caractéristiques ressortent : le canal est solidement implanté dans une tranchée taillée dans le rocher dans laquelle le béton a été coulé, lui servant ainsi de coffrage ; le cintre de la voute a laissé la trace des trois planches qui le formaient, et le rebord du piédroit qui permettait d'installer ce cintre.

\section{LA ROUTE DE GRASSE}

I.apueduc est supposé passer le long de la lagane (faubourg d'Antibes), à travers les serres Allard, enfin contourner l'église Notre-Dame d'Entrevignes et traverser la voie ferrée non loin du pont des Marseillais. La traversée des serres Allard a été vérifiée à l'occasion d'une récente opération de sauvetage (août 2004) due à un programme immobilier à cet emplacement. Deux sondages ont pu être pratiqués en bordures 


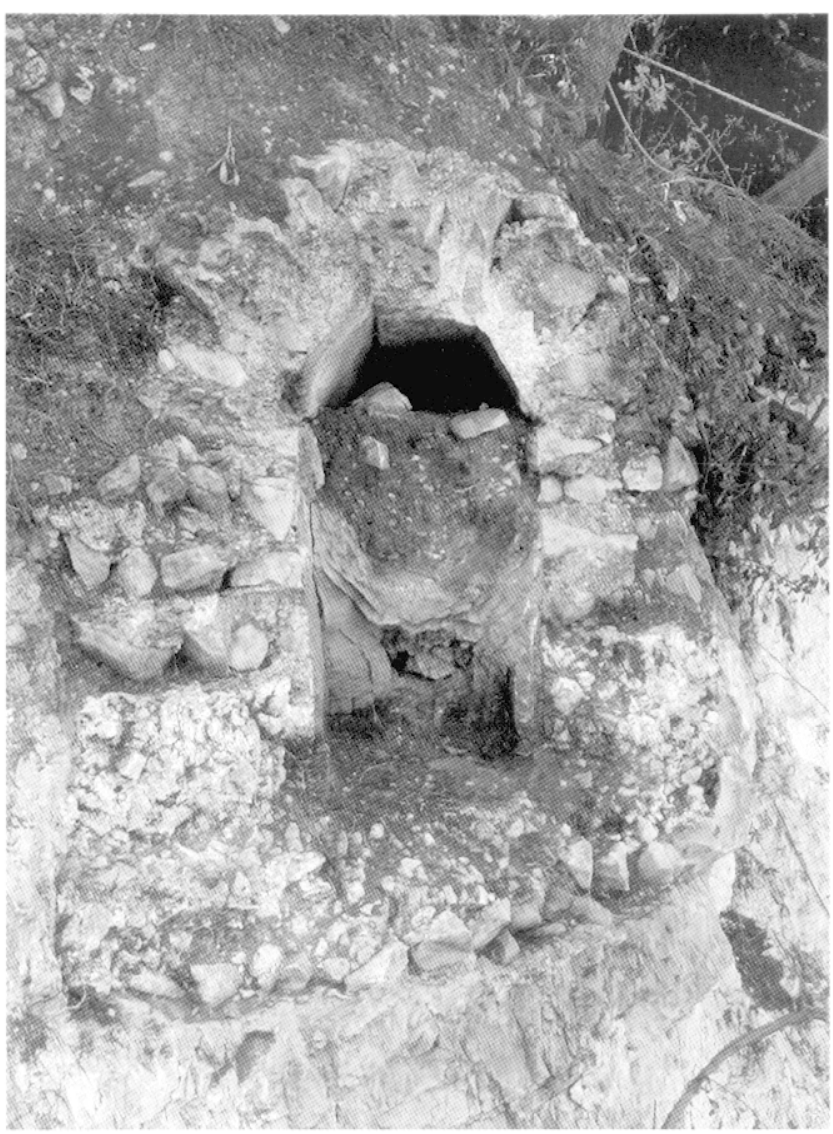

Fig. 27 - Aqueduc d'Antipolis, coupe du canal du chemin du Puy, résidence les Comores (cliché P. Garczynski, CNRS).

nord-ouest et sud-est du terrain avec l'aide des engins de terrassement mis à notre disposition par l'aménageur; ils ont permis d'observer et de relever le canal conservé jusqu'aux piédroits.

\section{ANTIBES}

Dans la ville d'Antibes, les vestiges signalés par J. Arazi étaient situés à l'angle de la rue Thiers et du boulevard Dugommier, ainsi qu'à l'angle de la place du Général-de-Gaulle et de la rue Aristide-Briand. P. Cosson situe l'extrémité de l'aqueduc sur la butte de la Poudrière ou de la citadelle, où se trouvait, jusqu'au $\mathrm{XIX}^{\mathrm{c}}$ s., un réservoir d'origine supposée romaine ${ }^{22}$.

22. I.e tronçon de l'aqueduc compris entre la porte de France et la citadelle a certainement été détruit lors de la construction des remparts à la fin du XV'II" et au début du XI'III" s., comme d'ailleurs le laisse entendre J. Arazi (voir introduction). Il ne faut pas perdre de vue que cette ligne de fortifications a remplacé un rempart antérieur, rectiligne, en rive gauche du Laval, venant s'appurer sur un bastion et sur une première citadelle. Ce rempart est représenté sur le Plan du siège dAntibes par les troupes du duc de Savoie en 1592, conservé aux archives de Turin. Enfin, si quelques restiges subsistaient éventuellement dans cette zone après l'édification des fortifications du XVT' $\mathrm{s}$., leur arasement à la fin du XIX" s. - remparts, bastions, poudrière et citadelle - a vraiscmblablement supprimé tout espoir de nouvelles découvertes.

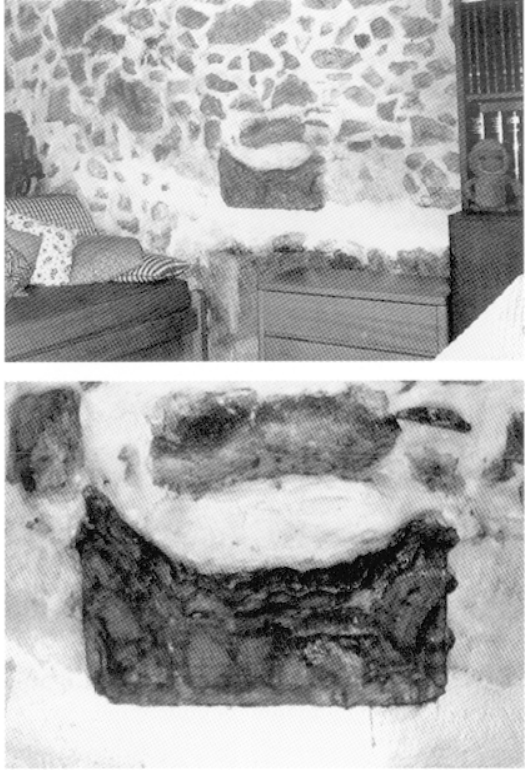

Fig. 28 - Aqueduc d'Antipolis, rue du Haut-Castelet : bloc de concrétion visible dans un mur à l'intérieur d'une maison; mur et canal reposent sur le rocher (clichés P. Garczynski, CNRS).

En 2001, une découverte d'un grand intérêt permet cependant d'émettre une nouvelle hypothèse. Le site se trouve être une maison de la rue du Haut-Castelet, sans doute à une centaine de mètres de l'extrémité de l'aqueduc : la présence du canal est manifestée par un bloc de concrétion de fond, en gobelet, saillant dans le mur d'une pièce, la maçonnerie récente qui a recouvert le reste du canal ayant épargné ce bloc (fig. 28). Les maisons de ce quartier sont installées directement sur le rocher et résultent, en fait, de la transformation progressive des cabanons de jardin qui se trouvaient là autrefois.

Ainsi le fait qu'il s'agisse du point le plus à l'aval connu avec certitude remet en cause l'arrivée de l'aqueduc dans l'agglomération d'Antipolis. Le château d'eau ou le bassin de répartition ne peuvent être localisés sur la butte de la citadelle, arasée lors de la démolition des fortifications d'Antibes ; il semblerait maintenant que l'aqueduc se dirige vers le castrum, ce que l'altitude du fil d'eau sur ce dernier site rend possible, mais il devait alors franchir la dépression située au sud-est de l'enceinte. L'hypothèse d'une arrivée à la tour de la Tourraque, plusieurs fois émise précédemment, retrouve ainsi une certaine crédibilité.

Les citernes romaines, entre la cathédrale et le château, sont bien situées sur le castrum, mais une visite dans la plus grande ne nous a pas révélé d'ouverture en partic haute pouvant représenter une arrivée d'eau.

\section{LES CARACTÉRISTIQUES DE L'OUVRAGE}

Bien que moyen par ses dimensions (section du canal et longueur), cet aqueduc présente un exemple des différents sites que l'on peut rencontrer sur d'autres ourrages plus impor- 
tants : canal bâti et enterré, canal taillé dans la roche, canal en tunnel ou tranchée profonde, canal à forte pente, bassin de tranquillisation, ponts et ponceau, bassin de décantation.

\section{LE CANAL}

La tranchée de fondation du conduit est généralement taillée dans la roche calcaire, de sorte que le fond en mortier de chaux soit moulé dans le substrat rocheux sur un hérisson ; la tranchée faisant office de banche. Sur le béton moulé sont montés les quatre assises de piédroits, puis la voûte (voir supra).

À l'approche d'un pont, le canal enterré passe en surface et doit présenter un parement extérieur ; celui-ci fait un angle de $37^{\circ}$ par rapport à la verticale. On ne connaît pas d'autre exemple, semble-t-il, de cette technique et nous sommes tentés de l'expliquer par un effet conjugué de solidité (effet de contrefort) et d'économie (moins de maçonnerie).

Le mortier de chaux utilisé sur les différents sites étudiés présente une grande variété de composition et de qualité. C'est ainsi que les agrégats, seuls composants pouvant être identifiés à l'oeil nu, vont du tuileau broyé plus ou moins finement au gravier roulé ou concassé, de granulométrie très variable. Au vu des différences de comportement des mortiers présents sur les sondages réalisés, il semble que leur qualité ait été variable.

Les moellons sont de type "en grain de maïs ", c'est-à-dire de forme trapézoïdale ou pointue, ainsi le travail de taille est réduit (pas de taille soignée pour les faces de jointoiement) et le mortier pénètre mieux dans les intervalles, assurant une meilleure cohésion entre le parement et le blocage intérieur donc une plus grande solidité de l'ensemble.

Les piédroits observés sont régulièrement formés de quatre assises de moellons et surmontés par un lit d'attente souvent légèrement incliné vers l'intérieur pour recevoir la voûte (Trois-Moulins).

En général il n'y a pas de mortier d'étanchéité interne : le premier dépôt de concrétion sur les parois en faisait sans doute office. L'exception concerne le pont à une arche.

La voûte est constituée de pierres peu taillées ou de forme naturellement allongée disposées en claveaux, ce qui incite à la définir comme sommairement clavéc. Par endroits l'abondance de mortier, notamment dans les cas où les planches de coffrage (en général trois) ont laissé leur empreinte, et un certain désordre dans la disposition des pierres ont pu quelques temps nous faire penser à une voûte concrète. L'extrados de la voûte est couvert d'une épaisse couche de mortier assurant son étanchéité.

\section{HYDRAULIQUE}

Les concrétions carbonatées constituent les "archives" du fonctionnement hydraulique d'un aqueduc. Dans ce cas, les dimensions relativement modestes du canal ainsi que l'absence de regards de visite interdisant toute opération de grattage des dépôts ont permis à ces "archives " d'être complètes et intactes aux endroits où la voûte n'a pas été démontée ou détruite.

\section{CIRCULATION DE L'EAU ET DÉBIT}

L'écoulement de l'eau dans un aqueduc romain est en général de type gravitaire à surface libre et ce n'est qu'exceptionnellement qu'il peut fonctionner en conduite forcée. Nous nous en tiendrons à une estimation du débit de l'aqueduc à sa mise en eau; pour cela la relation de Manning-Strickler est utilisée avec toute la prudence qu'il convient d'apporter à ce type de calcul (Viollet et al., 1998).

L'estimation proposée a été réalisée en deux points du canal : au niveau du site Euréka sur la branche de la Bouillide (4570 $\mathrm{m}^{3} /$ jour) et à l'arrivée dans l'agglomération sur le site du Haut-Castelet, sur la branche commune ( $5050 \mathrm{~m}^{3} / \mathrm{jour}$ ). Sur le premier site ont pu être évalués ou mesurés les paramètres du débit; en revanche, sur le second, la pente n'étant pas mesurable, la valeur adoptée est celle de la pente moyenne entre le précédent site connu et celui-ci.

Ces estimations, malgré l'incertitude qui leur est attachée, entraînent deux remarques: la différence des débits sur ces deux sites montre que les deux captages ont peut-être été utilisés dès l'origine, ce que propose également l'étude chimique des concrétions (voir infra). La différence quantitative de ces débits ne peut pas être utilisée pour estimer le débit de la branche de la Valmasque, car entre les deux sites considérés, les fuites externes du canal, matérialisées par des amas concrétionnés importants sur les parements des ponts, signalent une perte d'eau non négligeable.

\section{CONCEPTION HYDRAULIQUE DU CANAL}

L'observation de la hauteur de la première lamine des concrétions, chaque fois que cela a été possible, indique que lors de la mise en eau le niveau ne dépassait pas la partie en béton moulé. Les piédroits en petit appareil d'opus vittatum ne sont d'ailleurs revêtus d'aucun enduit d'étanchéité. Ce n'est que le dépôt ultérieur et progressif de concrétions abondantes qui a fait monter le niveau de l'eau pour finir par atteindre l'intrados de la voûte.

Il est intéressant de noter que les dimensions du béton moulé respectent les proportions pour que le débit d'un canal soit maximal: la hauteur d'eau est égale à la moitié de la largeur (sites Euréka et Comores : h. $=22 \mathrm{~cm} ; \mathrm{l} .=45 \mathrm{~cm}$ ).

\section{PERTE RAPIDE D'AlTITUDE}

Comme on a pu l'observer (descente INPI) un tronçon de l'aqueduc est sensiblement perpendiculaire aux courbes de niveau afin de perdre rapidement de l'altitude. Les concepteurs araient à leur disposition trois techniques pour réaliser une perte d'altitude : ils bâtissaient soit un canal à forte pente et à fond plat, soit un canal à forte pente et à fond en escalier, soit enfin pour des dénivelées importantes un canal composé de tronçons à pente faible reliés par des puits dans lesquels l'eau chutait de plusicurs mètres. Ici, c'est la première solution qui a été adoptée et le canal qui présente deux pentes différentes semble suive le profil du terrain antique.

Pour diminuer la vitesse et la turbulence de l'eau au bas d'une telle descente rapide, il était habituel de construire un 


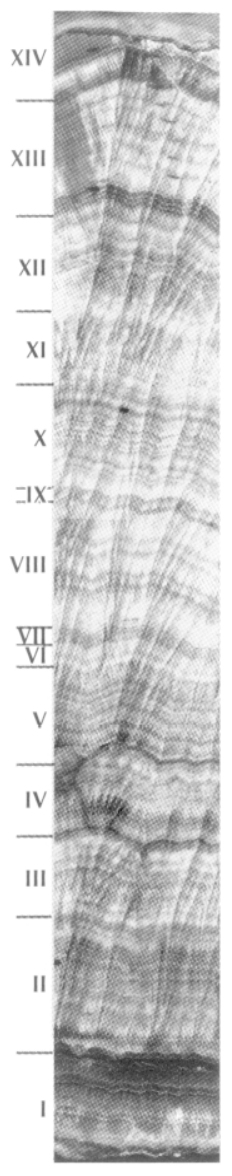

1 valeurs du périodogramme

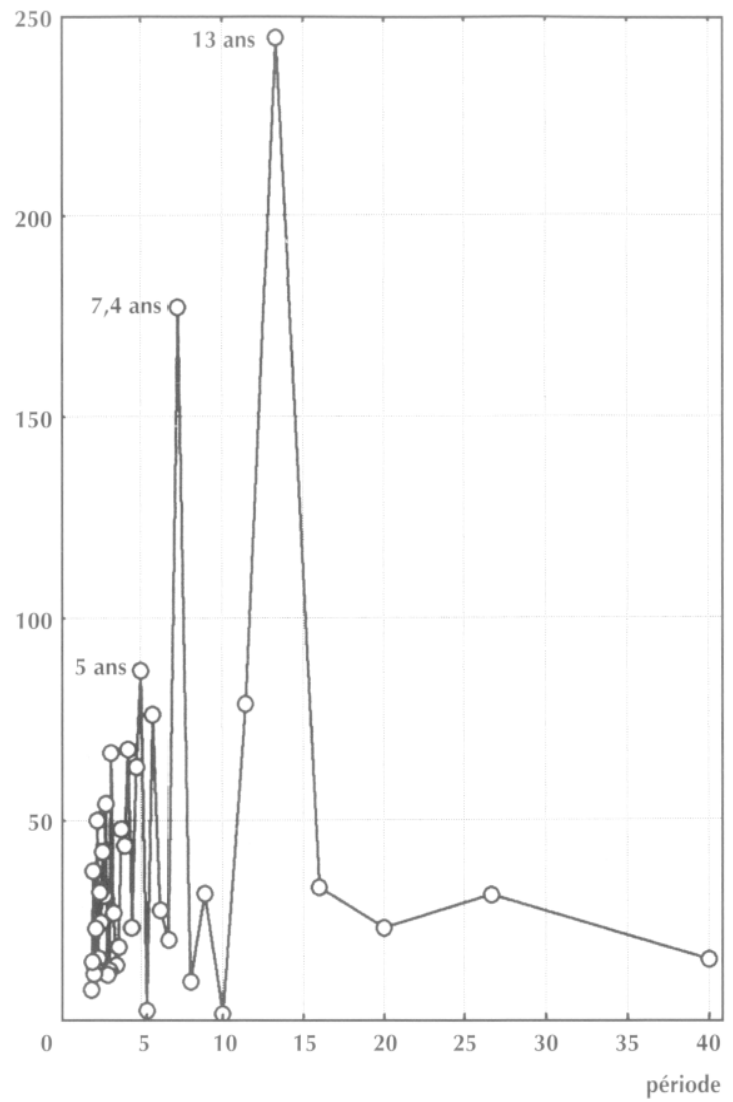

2
Fig. 29 - Aqueduc d'Antipolis : 1, section de laminite étudiée (14 faisceaux, 81 doublets de lamines; $h .: 26 \mathrm{~cm}) ; 2$, analyse spectrale (cliché et I)AO M. Dubar, (NRS). bassin de tranquillisation, que l'exploration au géoradar a permis de repérer.

\section{SYSTÈME CONCRÉTIONNAIRE}

Deux types d'études ont été engagées sur des concrétions internes prélevées en différents points du canal, certains essais étant toujours en cours. Les premiers travaux ont consisté à étudier l'agencement des lamines et les seconds à réaliser des microanalyses isotopiques des différentes lamines.

\section{AGENCEMENT DES LAMINES}

Cette étude a été menée sur un bloc épais de $27 \mathrm{~cm}$, prélevé sur le chantier Dostoïevski-Lefèbvre situé à quelques centaines de mètres en aval du tronçon en forte pente. Cette forte déclivité a favorisé l'agitation, le dégazage et donc la sursaturation des eaux et a ainsi provoqué des dépôts qui sont spécifiques de cette zone: il s'agit d'un concrétionnement fortement calcitisé. Ces concrétions représentent les premières années de fonctionnement de cette branche de l'aqueduc.

La série étudiéc comprend 81 doublets de lamines, alternativement claires et sombres, traditionnellement considérées comme saisonnières, répartis en 14 faisceaux (fig. 29).
L'analyse spectrale (Transformée de Fourier) réalisée sur l'épaisseur des doublets fait ressortir deux pics très énergétiques à 13 et 7,4 doublets et des pics moins robustes à de très hautes fréquences (à 5 doublets en particulier). Rapportées à des durées temporelles, ces fréquences sont bien connues par les climatologues et caractérisent l'activité solaire pour la première, l'oscillation nord-atlantique pour la seconde (Plaut et al., 1995). La présence de ces fréquences significatives permet d'assurer le caractère saisonnier des lamines et l'annualité du doublet. Ainsi, la concrétion étudiée correspondrait à une durée de 80 ans environ. Comme cette concrétion de $27 \mathrm{~cm}$ représente moins de la moitié du concrétionnement total de l'aqueduc, nous pouvons dire que la durée du fonctionnement de l'aqueduc serait de l'ordre de 160 ans.

\section{ANALYSES PHYSICO-CHIMIQUES ET ISOTOPIQUES}

Ces analyses, effectuées par S. Bonté ${ }^{23}$, ont porté sur trois prélèvements : l'un concernant la branche de la Bouillide et les deux autres la branche commune. Elles se basent sur la composition isotopique des lamines : ${ }^{13} \mathrm{C}$ et ${ }^{18} \mathrm{O}$. Bien que méritant

23. Doctorante, laboratoire d'hydrogéologie, EA 2665, LFR des sciences exactes et naturelles, université d'drignon et des pays de Vaucluse. 
d'être poursuivie et complétée, en particulier par des analyses isotopiques et chimiques des eaux des deux captages si cela est encore possible, une des conclusions préliminaires intéressantes est que les concrétions analysées peuvent être contemporaines, c'est-à-dire que les deux captages pourraient avoir été utilisés simultanément dès la mise en service de l'aqueduc.

\section{DATATIONS}

Les sondages n'ont jamais fourni de matériel pouvant aider à une datation de l'ourrage. Seule l'archivolte plate, détail architectural peu courant qui orne les arcs des ponts, permet de supposer que l'aqueduc est contemporain de celui de Fréjus (seconde moitié du $\mathrm{I}^{\mathrm{er}} \mathrm{s}$. ou début du $\mathrm{II}^{\mathrm{e}}$ s.) puisqu'on $\mathrm{y}$ retrouve ce même élément, également présent dans les thermes de Cimiez. On convient donc qu'il s'agit d'une particularité régionale, bien qu'on en connaisse quelques autres exemples en Italie, notamment à Herculanum et sur l'aqueduc de Minturno. Cela ne nous permet cependant pas de proposer une date précise.

On possède également peu d'indices permettant de comprendre les causes de destruction. Les fuites, attestées par les dépôts importants de concrétions sur les flancs des ponts, tendent à indiquer que des fissures s'étaient produites dans les parties aériennes du canal et que l'aqueduc connaissait des problèmes structurels. Enfin la section du canal ne permettant pas le passage d'un homme, l'aqueduc était donc voué à un fonctionnement sans entretien. Mais le cas du pont du Goa et les constatations qui ont pu être faites sur la pile IV et la culée aval permettent une hypothèse : la pile IV est affectée d'une inclinaison vers le nord-ouest, tandis que la culée aval, fendue du haut en bas par une large fissure, accuse, elle, une inclinaison vers le sud; des tassements différentiels opposés de ces deux éléments ont pu provoquer des fissures dans l'arche qui, se répercutant sur le canal, auraient engendré des fuites; les fissures finissant par provoquer la rupture et l'effondrement de l'arche.

Les vestiges au sol sont aussi recouverts d'importantes masses de concrétions, ce qui permet d'étendre cette hypothèse à plusieurs arches. Le pont du Fugueiret (à unc arche), lui aussi recouvert d'une énorme couche de concrétions, s'est également effondré. Pour le pont de la Valmasque en revanche, aucune trace de concrétions externes témoignant d'une fuite n'apparait, la construction n'aurait donc pas subi de déformation: peut-être est-ce pour cela qu'il est encore debout.
À côté de cette hypothèse d'une destruction causée par des imperfections dans la construction, on peut en proposer d'autres qui ne sont d'ailleurs pas forcément exclusives: campagnes militaires qui ont marqué l'histoire mouvementée d'Antibes, ville frontière ; effets des tremblements de terre dans une zone à sismicité non négligeable; enfin action de crues violentes des cours d'eau traversés.

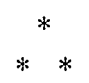

Malgré l'existence de vestiges apparents et un accès relativement facile sur une grande partie du tracé, l'aqueduc de la Bouillide était resté mal connu et de nombreuses erreurs persistaient à son sujet.

Nous l'avons étudié comme un monument complet, en l'analysant dans son entier malgré les destructions dont il a été l'objet, y compris ces dernières années. Notre étude a profité d'opportunités favorables comme le réaménagement de la gare autoroutière d'Antibes qui a permis la fouille et l'étude de l'ouvrage le plus important de l'aqueduc, le pont à cinq arches sur le Goa. Elle a aussi contribué à l'enrichissement de la carte archéologique et à la protection de ce patrimoine ; en effet, la Commission régionale du patrimoine et des sites (CRPS) a donné, en 2001, un avis favorable au classement Monuments historiques des ouvrages d'art de l'aqueduc (les trois ponts) et décidé l'inscription de toute la partie conservée, du captage à la colline des Croûtons.

Notre connaissance de l'ouvrage a progressé dans de nombreux domaines: cartographie détaillée et localisations précises s'appuyant sur les observations et les relevés de terrain, étude architecturale des ouvrages d'art, étude des bâtis en général et des techniques de construction, relevé des dépôts carbonatés et amorce de leur étude. La comparaison avec l'aqueduc de Fréjus et les thermes de Cimiez fait apparaître des particularités régionales, dont l'étude mériterait d'être approfondie.

\section{Nota bene}

Nous remercions pour leur aide E. Rouvière, A. Metz, Y. et M. Grout de Beaufort, G. Rivière de Vauguérin, M. Lanotte, G. Salacrou, G. Debergue et P. Martin (CG), B. Suau (Scetauroute), le commandant R. Blancardi (pompiers d'Antibes), E. Hermand et P.-Y. Delpech (Telluris), C. Carle (Espace vert entreprise); les municipalités d'Antibes, de Vallauris et de Valbonne, le Conseil général des AlpesMaritimes, le Service départemental de l'architecture et du patrimoine et le Service régional de l'archéologie. 